\title{
Long-Range Dependence, Fractal Processes, and Intra-Daily Data
}

\author{
Wei Sun \\ Institute for Statistics and Mathematical Economics \\ University of Karlsruhe, Germany \\ Svetlozar Rachev* \\ Institute for Statistics and Mathematical Economics \\ University of Karlsruhe, Germany \\ \& \\ Department of Statistics and Applied Probability \\ University of California, Santa Barbara, USA \\ Frank J. Fabozzi \\ Yale School of Management, USA
}

\begin{abstract}
With the availability of intra-daily price data, researchers have focused more attention on market microstructure issues to understand and help formulate strategies for the timing of trades. The purpose of this article is to provide a brief survey of the research employing intra-daily price data. Specifically, we review stylized facts of intra-daily data, econometric issues of data analysis, application of intra-daily data in volatility and liquidity research, and the applications to market microstructure theory. Long-range dependence is observed in intra-daily data. Because fractal processes or fractional integrated models are usually used to model long-range dependence, we also provide a review of fractal processes and long-range dependence in order to consider them in future research using intra-daily data.
\end{abstract}

\section{Introduction}

With the adoption of electronic trading and order routing systems, an enormous quantity of trading data in electronic form is now available. A complete data set of transactions recorded and their associated characteristics such as transaction time, transaction price, posted bid/ask prices, and volumes are

\footnotetext{
${ }^{*}$ Corresponding author: Prof. Dr. S. Rachev, Institute for Statistics and Mathematical Economics, Uni-Karlsruhe, Postfach 6980, 76128 Karlsruhe, Germany. Email: rachev@statistik.uni-karlsruhe.de. W. Sun's research was supported by grants from the Deutschen Forschungsgemeinschaft. S. Rachev's research was supported by grants from Division of Mathematical, Life and Physical Science, College of Letters and Science, University of California, Santa Barbara, and the Deutschen Forschungsgemeinschaft.
} 
provided. These data are gathered at the ultimate frequency level in the financial markets and usually referred to as intra-daily data or high-frequency data.

There is no standardization of the term intra-daily data adopted by researchers in the market microstructure area. In this literature, there are several descriptions for intra-daily data. Hasbrouck (1996) mentions microstructure data or microstructure time series. Engle (2000) uses the term ultrahigh frequency data to describe the ultimate level of disaggregation of financial time series. Alexander (2001) describes high-frequency data as real time tick data. Gourieroux and Jasiak (2001) use the expression tick-by-tick data while Tsay (2002) writes "high frequency data are observations taken at fine time intervals." In this article, the term intra-daily data will be used interchangeably with the other terms used by market structure researchers as identified above.

A summary of the literature covering intra-daily data research has been provided in several publications from 1996 to 2004. Hasbrouck (1996) provides an overview of the statistical models of intra-daily data are used for explaining trading behavior and market organization. Goodhart and O'Hara (1997) summarize many important issues connected with the use, analysis, and application of intra-daily data. Madhavan (2000) provides a survey covering the theoretical, empirical, and experimental studies of market microstructure theory. Ghysels (2000) reviews the econometric topics in intra-daily data research, identifying some challenges conforting researchers. In a collection of papers edited by Goodhard and Payne (2000), several works based the intra-daily data from foreign exchange market are presented. Gwilym and Sutcliff (1999), Bauwens and Giot (2001), and Dacorogna et al. (2001) provide detailed coverage of econometric modeling as it pertains to the empirical analysis of market microstructure. The survey by Easley and O'Hara (2003) is presented in terms of microstructure factors in asset pricing dynamics. Engle and Russel (2004) highlight econometric developments pertaining intra-daily data. Harris (2003) and Schwartz and Francioni (2004) provide insights into market microstructure theory based on discussions with practitioners, focusing less on theoretical and econometric issues associated with intra-daily data.

As the full record of every movement in financial markets, intra-daily data offer the researcher or analyst a large sample size that increases statistical confidence. The data can reveal events in the financial market that are impossible to identify with low frequency data. While in some sense, intradaily data might be regarded as the microscope used for studying financial markets, these data have broader interest in econometric modeling and market microstructure research. Some other implications of intra-daily data are being identified in the literature. A fundamental property of intra-daily data is "observations can occur at varying random time intervals" (see, Ghysels (2000)). Other fundamental statistical properties that have been observed by researchers to characterize intra-daily data are fat tails, volatility clustering, daily and weekly patterns, and long-range dependence (see, for example, Dacorogna et al. (2001)). Based on these observed stylized factors, time series models and market microstructure models have been built. Time series models capture the statistical properties of financial data, while market microstructure models explain trading behavior and market organization.

Besides explaining market microstructure theory, intra-daily data has been used in studying various topics in finance. Some examples are risk management (e.g., Beltratti and Morana (1999)), model evaluation (e.g., Gençay et al.(2002), and Bollerslev and Zhang (2003)), market efficiency test (e.g., Hotchkiss and Ronen (2002)), electricity price analysis (e.g., Longstaff and Wang (2004)), information 
shocks to financial market (e.g., Faust et al. (2003) and Adams et al. (2004)), and financial market anomalies (e.g., Gleason et al. (2004)).

Any survey of a field with extensive research must be necessarily selective in its coverage. This is certainly the case we faced in preparing this review on research of intra-daily data, fractal processes, and long-range dependence. In this paper, we provide an aerial view of the literature, attempting to combine the research of fractal processes and long-range dependence together with the use of intra-daily data.

We organized the paper as follows. In Section 2, we discuss the stylized facts of intra-daily data observed in financial markets. Computer implementations in studying intra-daily data are the subject of Section 3. In Section 4, we review the major studies on intra-daily data analyzed in financial markets, running the risk that we have overlooked some important studies somewhere in hyperspace that we failed to uncover or published while this work was in progress. In Section 5, we discuss the anaylsis of long-range dependence, then introducing two fractal processes, (fractional Gaussian noise and fractional stable noise) in Section 6. Our concluding remarks appear in Section 7.

\section{Stylized Facts of Financial Intra-daily Data}

In data analysis, an important task is to identify the statistical properties of the target data set. Those statistical properties are referred to as stylized factors. Stylized factors offer building blocks for further modeling in such a way so as to encompass these statistical properties. Being full record of market transactions, intra-daily data have properties that have been observed. In this section, some stylized factors of intra-daily data will be reviewed.

\subsection{Random Durations}

For intra-daily data, irregularly spaced time intervals between successive observations is the salient feature compared with classical time series data (see, for example, Engle (2000) and Ghysels (2000)). Given time points equally spaced along the time line, for intra-daily data, at one time point, there might be no observation or several observations. In intra-day data, observations arrive at random time. This causes the duration between two successive observations not to be constant in a trading day. For

a time series, if the time space (duration) is constant over time, it is an equally spaced time series or homogeneous time series. If the duration varies throught time, it is an unequally spaced time series, i.e., the inhomogeneous time series, see Dacorogna et al. (2001).

\subsection{Distributional Properties of Returns}

Many techniques in modern finance rely heavily on the assumption that the random variables under investigation follow a Gaussian distribution. However, time series observed in finance often deviate from the Gaussian model, in that their marginal distributions are found to possess heavy tails and are possibly asymmetric. Bollerslev et al. (1992) mentions that intra-daily data exhibit fatter tails in the unconditional return distributions and Dacorogna et al. (2001) confirms the exhibition of heavy tails in intra-daily returns data. 
In such situations, the appropriateness of the commonly adopted normal distribution assumption for returns is highly questionable in research involving intra-daily data. It is often argued that financial asset returns are the cumulative outcome of a vast number of pieces of information and individual decisions arriving almost continuously in time. Hence, in the presence of heavy tails, it is natural to assume that they are approximately governed by a non-Gaussian stable distribution, see Rachev and Mittnik (2000), and Rachev et al. (2005). Marinelli et al. (2000) first model the heavy tailedness in intra-daily data. Mittnik et al. (2002) point out that other leptokurtic distributions, including Student's $t$, Weibull, and hyperbolic, lack the attractive central limit property. Sun et al. (2006a) confirm the findings of heavy tailedness in intra-daily data.

Wood et al. (1985) present evidence that stock returns are not independently and identically distributed. They find that the distributions are different for the return series in the first 30 minutes of the trading day, at the market close of the trading day, and during the remainder of the trading day.

\subsection{Autocorrelation and Seasonality}

The study by Wood et al. (1985), one of the earliest studies employing intra-daily data, finds that the trading day return series is non-stationary and is characterized by a low-order autoregressive process. For the volatility of asset returns, autocorrelation has been documented in the literature (see among others, Engle (1982), Baillie and Bollerslev (1990), Bollerslev et al. (1992), Hasbrouck (1996), and Bollerslev and Wright (2001)). The existence of negative first-order autocorrelation of returns at higher frequency, which disappears once the price formation is over, has been reported in both the foreign exchange market and equity market. The explanation for the finding of negative autocorrelation of stock returns observed by researchers is due to by what is termed the bid-ask bounce. According to the bid-ask bounce explanation, the probability of a trade executing at the bid price and then being followed by a trade executing at the ask price is higher than a trade at the bid price followed by another trade at the bid price (see Alexander (2001), Dacorogna et al. (2001), and Gourieroux and Jasiak (2001)).

Many intra-daily data display seasonality. By seasonality, we mean periodic fluctuations. Daily patterns in the trading day have been found in the markets for different types of financial assets (see, Jain and Joh (1988), McInish and Wood (1991), Bollerslev and Domowitz (1993), Engle and Russel (1998), Andersen and Bollerslev (1997), Bollerslev et al. (2000), and Veredas et al. (2002)). One such trading pattern is the well-known "U-shape" pattern of daily trading (see, Wood et al (1985), Ghysels (2000), and Gourieroux and Jasiak (2001)). This trading pattern refers to the observation in a trading day that trade intensity is high at the beginning and at the end of the day, and trading durations increase and peak during lunch time. As a result, return volatility exhibits a U-shape where the two peaks are the beginning and the end of a trading day, with the bottom approximately during the lunch period. Hong and Wang (2000) confirm the U-shape patterns in the mean and volatility of returns over trading day. They find that around the close and open there exist higher trading activity, the returns of open-to-open being more volatile than that of close-to-close. Besides the intra-day pattern, there exists a day-of-week pattern evidenced by both lower returns and higher volatility on Monday. 


\subsection{Clustering}

Many financial time series display volatility clustering. It is observed that large returns are followed by more large returns and small returns by more small returns. Equity, commodity, and foreign exchange markets often exhibit volatility clustering at higher frequency. Volatility clustering becomes pronounced in intra-daily data (see, for example, Alexander (2001), Haas et al. (2004)). Besides volatility clustering, intra-daily data exhibit quote clustering and duration clustering. Quote or price clustering is the preference for some quote/prices over others. Duration clustering is the long and short durations tend to occur in clusters (see, for example, Bauwens and Giot (2001), Chung and Van Ness (2004), Engle and Russell (1998), Feng et al. (2004), Huang and Stoll (2001), and Sun et al.(2006b)).

\subsection{Long-range Dependence}

Long-range dependence or long memory (sometimes also referred to as strong dependence or persistence) denotes the property of time series to exhibit persistent behavior. (A more precise mathematical definition will be provided in Section 5.) It is generally believed that when the sampling frequency increases for financial returns, long-range dependence will be more significant. Marinelli et al. (2000) propose subordinated modeling to capture long-range dependence and heavy tailedness. Several researchers, focusing both on theoretical and empirical issues, discuss long-range dependence. Doukhan et al. (2003), Robinson (2003), and Teyssiére and Kirman (2006) provide an overview of the important contributions to this area of research. Investigating the stocks comprising the German DAX, Sun et al.(2006a) confirm that long-range dependence does exist in intra-daily return data. We provide a more detailed discussion of long-range dependence in Section 5.

\section{Computer Implementation in Studying Intra-daily Data}

\subsection{Data Transformation}

Being a full record of transactions and their associated characteristics, intra-daily data represent the ultimate level of frequency at which trading data are collected. The salient feature of such data is that they are fundamentally irregularly spaced. It is necessary to distinguish intra-daily data from high frequency data because the former are irregularly spaced while the latter are sometimes spaced by aggregating to a finer fixed-time interval. In order to clarify the time interval, it is useful to refer to the data by its associated time interval. For example, if the raw intra-daily data have been aggregated to create an equally-spaced time series, say five minutes interval, then the return series is referred to as the "5 minutes intra-daily data". In order to clarify the characteristic of the interval between data points, Dacorogna et al. (2001) propose employing a definition of homogeneous and inhomogeneous time series. The irregularly spaced time series is called an inhomogeneous time series while the equally spaced one is called a homogeneous time series. One can aggregate inhomogeneous time series data up to a specified fixed time interval in order to obtain a corresponding homogeneous version. Naturally, such aggregation will either lose information or create noise, or both. For example, if the observations in the original data are more than that in the aggregated one, some information will be lost. If the observations in the orginal data are much less than that in the aggregated one, noise will be generated. Data interpolation is required to create aggregated time series. Obviously, different interpolation methods lead to different 
results. This leads not only to the loss of information but also the creation of noise due to introducing errors in the interpolation process. Engle and Russel (1998) argue that in the aggregation of tick-by-tick data to some fixed time interval, if a short time interval is selected, there will be many intervals having no new information and, as a result, heteroskedasticity will be introduced; and if wide interval is chosen, microstructure features might be missing. Therefore, it is reasonable to keep the data at the ultimate frequency level (see Aït-Sahalia et al. (2005)).

Standard econometric techniques are based on homogeneous time series analysis. Applying analytical methods of homogeneous time series to inhomogeneous time series may produce unreliablity. That is the dichotomy in intra-daily data analysis: a researcher can retain the full information without creating noise but is challenged by the burden of technical complexity. Sometimes, it is not always necessary to retain the ultimate frequency level. In those instances, aggregating inhomogeneous intradaily data to a relatively lower but still comparably higher frequency level of homogeneous time series is needed. Wasserfallen and Zimmermann (1995) show two interpolation methods: linear interpolation and previous-tick interpolation. Given an inhomogeneous time series with times $t_{i}$ and values $\varphi_{i}=\varphi\left(t_{i}\right)$, the index $i$ identifies the irregularly spaced sequence. The target homogeneous time series is given at times $t_{0}+j \Delta t$ with fixed time interval $\Delta t$ starting at $t_{0}$. The index $j$ identifies the regularly spaced sequence. The time $t_{0}+j \Delta t$ is bounded by two times $t_{i}$ of the irregularly spaced series,

$$
\begin{gathered}
I=\max \left(i \mid t_{i} \leq t_{0}+j \Delta t\right) \\
t_{I} \leq t_{0}+j \Delta t>t_{I+1}
\end{gathered}
$$

Data will be interpolated between $t_{I}$ and $t_{I+1}$. The linear interpolation shows that

$$
\varphi\left(t_{0}+j \Delta t\right)=\varphi_{I}+\frac{t_{0}+j \Delta t-t_{I}}{t_{I+1}-t_{I}}\left(\varphi_{I+1}-\varphi_{I}\right)
$$

and previous-tick interpolation shows that

$$
\varphi\left(t_{0}+j \Delta t\right)=\varphi_{I}
$$

Dacorogna et al. (2001) point out that linear interpolation relies on the future information whereas previous-tick interpolation is based on the information already known. Müller et al. (1990) suggest that linear interpolation is an appropriate method for independent and identically distributed (i.i.d.) increments stochastic processes.

More advanced techniques have been adopted by some researchers in order to find sufficient statistical properties of data but at the same time retaining the inhomogeniety of time series. Zumbach and Müller (2001), for example, propose a convolution operator to transform the original inhomogeneous time series to a new inhomogeneous time series in order to get more sophisticated quantities. Newly developed techniques such as the wavelet method have been adopted to analyze intra-daily data. For example, Gençay et al $(2001,2002)$ employed a wavelet multiscaling method to remove intra-daily seasonality in five-minute intra-daily data of foreign exchange.

As mentioned in Section 2, intra-daily data exhibit daily patterns. Several methods of data adjusting have been adopted in empirical analysis in order to remove such pattern (see, Engle and Russell (1998), Veredas et al. (2002), Bauwens and Giot (2000, 2003), and Bauwens and Veredas (2004)). 


\subsection{Data Cleaning}

In order to improve the quality of data, data cleaning is required to detect and remove errors and inconsistencies from the data set. Data quality problems are the result of misspelling during data entry, missing information, and other kinds of data invalidity. There are two types of error: human errors and computer system errors (see Dacorogna et al. (2001)). When multiple data sources must to be integrated, for instance, pooling the data in each trading day together for one year, the need for data cleaning increases significantly. The reason is that the sources often contain redundant data in different representations. High quality data analysis requires access to accurate and consistent data. Consequently, consolidating different data representations and eliminating duplicate information become necessary.

The object of data cleaning is the time series of transactions information. Usually, the transactions information is "quote" or "tick" information. Each quote or tick in the intra-daily data set contains a time stamp, an identification code, and variables of the tick, such as bid/ask price (and volume), trade price (and volume), and locations. Intra-daily data might contain several errors that should be specially treated. Decimal errors occur when the quoting software uses cache memory so that it is failure to change a decimal digit of the quote. Test tick errors are caused by data managers' testing operation of sending artificial ticks to the system to check the sensitivity of recording. Repeated ticks are caused by data managers' test operation of letting the system repeat the last tick in the specified time intervals. Some errors occur when data managers copy the data or when a scaling problem occurs, see Dacorogna et al. (2001).

Coval and Shumway (2001) illustrate the existence of occasionally incorrect identification of the exact time. They use the data cleaning method to ensure that the time stamps on each tick were accurate and scaled to the second level. They introduce a method of summing up variables from 31 seconds past one minute to 30 seconds past the next minute to aggregate the tick to the minute level. Some detailed methods used in data cleaning are discussed in Dacorogna et al. (2001).

\section{Research on Intra-Daily Data in Finance}

\subsection{Studies of Volatility}

Volatility is one of the most important risk metric. Harris (2003) defines it as the tendency for prices unexpectedly changing. Volatility could be regarded as the market reaction to news reflected by price changing. He distinguishes fundamental volatility and transitory volatility. Fundamental volatility is caused by the endogenous variables which determine the value of trading instruments. Transitory volatility is due to trading activity by uninformed traders.

Volatility is not constant over the trading stage, but changes over time. Transitory volatility might occur in a very short time period before it converts to its fundamental value. The intra-daily data can observe both fundamental volatility and transitory volatility with sufficient statistical significance.

Engle (2000) adopts the GARCH model to the irregularly spaced ultra-high frequency data. Letting $d_{i}$ be the duration between two successive transactions and $r_{i}$ the return between transactions $i-1$ and 
$i$, then the conditional variance per transaction is:

$$
V_{i-1}\left(r_{i} \mid d_{i}\right)=\phi_{i}
$$

and the conditional volatility per unit of time is defined as,

$$
V_{i-1}\left(\frac{r_{i}}{\sqrt{d_{i}}} \mid d_{i}\right)=\sigma_{i}^{2}
$$

Then the connection between equations (5) and (6) can be established by $\phi_{i}=d_{i} \sigma_{i}^{2}$. The predicted variance conditional on past returns and durations is $E_{i-1}\left(\phi_{i}\right)=E_{i-1}\left(d_{i} \sigma_{i}^{2}\right)$. Using $\operatorname{ARMA}(1,1)$ with innovations $\varepsilon_{i}$, the series of return per unit of time is

$$
\frac{r_{i}}{\sqrt{d_{i}}}=a \frac{r_{i-1}}{\sqrt{d_{i-1}}}+\varepsilon_{i}+b \varepsilon_{i-1}
$$

If the current duration contains no information, the simple GARCH specification is used, and then

$$
\sigma_{i}^{2}=\omega+\alpha \varepsilon_{i-1}^{2}+\beta \sigma_{i-1}^{2}
$$

If durations are informative, Engle (2000) proposes an autoregressive conditional duration (ACD) model to define the expected durations. If the ACD model is

$$
\psi_{i}^{2}=h+m d_{i-1}+n \psi_{i-1}
$$

then the ultra-high frequency GARCH model is expressed as:

$$
\sigma_{i}^{2}=\omega+\alpha \varepsilon_{i-1}^{2}+\beta \sigma_{i-1}^{2}+\gamma_{1} d_{i}^{-1}+\gamma_{2} \frac{d_{i}}{\psi_{i}}+\gamma_{3} \xi_{i-1}+\gamma_{4} \psi_{i}^{-1}
$$

where $\xi_{i-1}$ is the long-run volatility computed by exponentially smoothing $r^{2} / d$ with a parameter 0.995 such that

$$
\psi_{i}=0.005\left(\frac{r_{i-1}^{2}}{d_{i-1}}\right)+0.995 \psi_{i-1}
$$

Alexander (2001) points out that a number of studies have shown that the aggregation properties of GARCH models are not straightforward. The persistence in volatility seems to be lower when it is measured using intra-day data than when measured using daily or weekly data. For example, fitting a GARCH $(1,1)$ to daily data would yield a sum of autoregressive parameter and moving average parameter estimates that is greater than the sum of that estimated from fitting the same GARCH process by using 2-day returns. In the heterogeneous ARCH (HARCH) model proposed by Müller et al. (1997), a modified process is introduced so that the squared returns can be taken at different frequencies. The return of $r_{t}$ of the $\operatorname{HARCH}(n)$ process is defined as follows,

$$
\begin{aligned}
r_{t} & =h_{t} \varepsilon_{t} \\
h_{t}^{2} & =\alpha_{0}+\sum_{j=1}^{n} \alpha_{j}\left(\sum_{i=1}^{j} r_{t-i}\right)^{2}
\end{aligned}
$$

where $\varepsilon_{t}$ is i. i. d. random variables with zero mean and unit variance, $\alpha_{0}>0, \alpha_{n}>0, \alpha_{j} \geq 0$, for $j=1,2, \ldots, n-1$. The equation for the variance $h_{t}^{2}$ is a linear combination of the squares of aggregated 
returns. Aggregated returns may extend over some long intervals from a time point in the distant past up to time $t-1$. The HARCH process belongs to the wide ARCH family but differs from all other ARCH-type processes in the unique property of considering the volatilities of returns measured over different interval size. Dacorogna et al. (2001) generalized the HARCH process. In equation (12), all returns considered by the variance equation are observed over recent interval ending at time $t-1$. This strong limitation is justified by its empirical successive observations, but a more general formula of the process with observation intervals ending in the past before $t-1$ can be shown as follows:

$$
\begin{aligned}
r_{t} & =h_{t} \varepsilon_{t} \\
h_{t}^{2} & =\alpha_{0}+\sum_{j=1}^{n} \sum_{k=1}^{j} \alpha_{j k}\left(\sum_{i=k}^{j} r_{t-i}\right)^{2}+\sum_{i=1}^{q} b_{i} h_{t-i}^{2}
\end{aligned}
$$

where

$$
\begin{aligned}
\alpha_{0}>0, \alpha_{j k} \geq 0, & \text { for } \quad j=1,2, \ldots, n, k=1,2, \ldots j \\
b_{j} \geq 0 \quad \text { for } \quad & i=1,2, \ldots, q
\end{aligned}
$$

The generalized process equation considers all returns between any pair of two time points in the period between $t-n$ and $t-1$. It covers the case of HARCH (all $\alpha_{j k}=0$ except some $\alpha_{j 1}$ ), as well as that of $\mathrm{ARCH}$ and GARCH (all $\alpha_{j k}=0$ except some $\left.\alpha_{j j}\right)$.

From historical data, realized volatility can be computed. Dacorogna et al. (2001) show the realized volatility as:

$$
v\left(t_{i}\right)=\left[\frac{1}{n} \sum_{j=1}^{n}\left|r\left(\Delta t ; t_{i-n+j}\right)^{p}\right|\right]^{1 / p}
$$

where $n$ is the number of return observations, and $r$ stands for the returns in the regularly spaced time intervals. $\Delta t$ is the return interval. Taylor and Xu (1997), Andersen and Bollerslev (1998), and Giot and Laurent (2004), among others, show that summing up intra-daily squared returns can estimate the daily realized volatility. Given that a trading day can be divided into $n$ equally spaced time intervals, and if $r_{i, t}$ denotes the intra-daily return of the $i$ th interval of day $t$, the daily volatility for day $t$ can be expressed as:

$$
\left[\sum_{i=1}^{n} r_{i, t}\right]^{2}=\sum_{i=1}^{n} r_{i, t}^{2}+2 \sum_{i=1}^{n} \sum_{j=i+1}^{n} r_{j, t} r_{j-i, t}
$$

Andersen et al. (2001a) show that if the returns have a zero mean and are uncorrelated, $\sum_{i=1}^{n} r_{i, t}^{2}$ is a consistent and an unbiased estimator of the daily variance. $\left[\sum_{i=1}^{n} r_{i, t}\right]^{2}$ is called the daily realized volatility since all squared returns on the right side of equation (16) can be observed when intra-daily data sampled over an equally spaced time interval are available. This is one method for modeling daily volatility using intra-daily data, and a method that has been generalized by Andersen et al. (2001a, 2001b). Another method is to estimate the intra-daily duration model on trade durations for a given asset. It is observed that longer durations lead to lower volatility and shorter durations lead to higher volatility and durations are informative, see Engle (2000) and Dufour and Engle (2000). Gerhard and Hautsch (2002) estimate daily volatility for an intra-daily duration model at which irregularly time spaced volatility has been used at the aggregated level. 
There are several articles that provide a detailed discussion of modeling volatility, see, for example, Andersen et al. (2005a, 2005b, 2006). Modeling and forecasting volatility based on intra-daily data has attracted a lot of research interest. Andersen et al. (2001) improve the inference procedures for using intra-daily data forecasts. Bollerslev and Wright (2001) propose a method to model volatility dynamics by fitting an autoregressive model to log-squared, squared or absolute returns of intra-daily data. They show that when working with intra-daily data, using a simple autoregressive model can provide a better prediction for forecasting future volatility than standard GARCH or exponential GARCH (EGARCH) models. They suggest that intra-daily data can be easily used to generate superior daily volatility forecasts. Blair et al. (2001) offer evidence that intra-daily returns provide much more accurate forecasts of realized volatility than daily returns. In the context of stochastic volatility models, Barndorff-Nielsen and Shephard (2002) investigate the statistical properties of realized volatility. Corsi et al. (2005) study the time-varying volatility of realized volatility. Using a GARCH diffusion process, Martens (2001) and Marten et al. (2002) point out that using intra-daily data can improve the out-of-sample daily volatility forecasts. Bollen and Inder (2002) use the vector autoregressive heteroskedasticity and autocorrelation (VARHAC) estimator to estimate daily realized volatility from intra-daily data. Andersen et al. (2003) propose a modeling framework for integrating intra-daily data to predict daily return volatility and return distribution. Thomakos and Wang (2003) investigate the statistical properties of daily realized volatility of futures contracts generated from intra-daily data. Using 5-minute intra-daily foreign exchange data, Morana and Beltratti (2004) illustrate the existence of structural breaks and long memory in the realized volatility process. Fleming et al (2003) indicate that the economic value of the realized volatility approach is substantial and deliver several economic benefits for investment decision making. Andersen et al. (2005a) summarize the parametric and non-parametric methods used in volatility estimation and forecasting.

\subsection{Studies of Liquidity}

A topic of debate in finance is the meaning of liquidity. Some market participants refer to liquidity as the ability to convert an asset into cash quickly, some define liquidity in terms of the low transaction cost, and some think high transaction activity is liquidity, see Easley and O'Hara (2003) and Schwartz and Francioni (2004). Schwartz and Francioni point out that a better approach for defining liquidity should be based on the attributes of liquidity. They define the depth, breadth, and resiliency as the dimensions of liquidity, while Harris (2003) identifies immediacy, width, and depth as the relevant attributes of liquidity. In the microstructure literature, the bid/ask spread proxies for liquidity, see Easley and O'Hara (2003). Schwartz and Francioni (2004) show that liquidity can be approximated by the trade frequency of an asset traded in the market. The frequency can be measured by the magnitude of short-term price fluctuation for such asset. Chordia et al. (2002) find that order imbalances affect liquidity and returns at the aggregate market level. They suggest using order imbalance as a proxy for liquidity.

Using bid/ask spread as a proxy for liquidity, Chung et al. (2001) compare the bid/ask spread on the NYSE and Nasdaq. They find that the average NYSE specialist spreads are significant smaller than the Nasdaq specialist spreads. Huang and Stoll (2001) report that dealer markets have relative higher spreads than auction markets. By comparing spreads in different markets, they found that the spreads on London Stock Exchange are larger than that for the same stocks listed on the NYSE and the spreads 
in the Nasdaq are larger than that on stocks listed on the NYSE. By checking the growth of electronic communication networks (ECNs) in Nasdaq, Weston (2002) confirms that the electronic trading system has the ability to improve the liquidity on the Nasdaq. Kalay et al. (2004) report that the opening is more liquid than the continuous trading stage. For small price changes and small quantities, there is a less elastic supply curve than demand curve.

From the perspective of liquidity providers, researchers usually use the order book data. Coughenour and Deil (2002) catagorize the specialist firms on the NYSE into two types: owner-specialist firms and employee-specialist firms. By investigating the influence of these two types of liquidity providers, they show that with similar trading costs, the owner-specialist firms have greater frequency of large trades and have greater incentive to reduce adverse selection costs. For employee-specialist firms, the stocks traded exhibit less sensitivity between change in quoted depth and quoted spreads. Meantime, these stocks show price stability at the opening. Peterson and Sirri (2002) investigate order submission strategies and find that limit orders perform worse than market orders involving the trading costs. But investors still prefer limit orders, suggesting that individual investors are less able to choose an optimal trading strategy.

\subsection{Studies of Market Microstructure}

Market microstructure refers to the description of trading processes for obtaining a better understanding of how prices adjust in order to reflect new information. Researchers in this field are typically willing to investigate the information contained in intra-daily data. The trading mechanisms and the price information flows are the major interest of market microstructure studies. Madhavan (2000) provides a good survey of market microstructure research based on four topics: price formation, market structure and design, transparency, and other applications.

Transaction costs, private information, and alternative trading systems have been investigated in the research on trading processes. Transaction costs are "market frictions" and reduce the trading frequency of investors. O'Hara (1995) and Madhavan (2000) demonstrate that private information will impact the investors' belief of the asset value. If investors possess private information, new information arrivals can be reflected by the order flows. The market structure might influence the size of trading costs. Different market structures have different functions for finding prices and matching buyers and sellers. The transparency of certain market structure also affects the functioning of market mechanisms, see Bollmfield and O'Hara (1999, 2000), O'Hara (1995), Spulber (1999), and Madhavan (2000).

How are prices determined in the financial market? As Madhavan (2000) notes, studying the market maker is the logical starting point for any analysis of market price determination. In market microstructure studies, inventory and asymmetric information are the factors that influence the price movement. Naik and Yadav (2003) examine whether equivalent inventories or ordinary inventories dominate the process of trading and pricing decisions by dealer firms. They find that ordinary inventories play the main role consistent with the decentralized nature of market making. Bollen et al. (2004) propose a new model based on intra-daily data to understand and measure the determinants of bid/ask spread of market makers. Using the vector autoregressive model, Dufour and Engle (2000) find that time durations between transaction impact the process of price formation. Engle and Lunde (2003) develop a bivariate model of trades and quotes for NYSE traded stocks. They find that high trade arrival rates, 
large volume per trade, and wide bid/ask spreads can be regarded as information flow variables that revise prices. They suggest that if such information is flowing, prices respond to trades more quickly.

In the study of market quality, much of the debate in the literature, according to Madhavan (2000), centers on floor versus electronic markets, and auctions versus dealer system. Using intra-daily data for Bund futures contracts, Frank and Hess (2000) compare the electronic system of the DTB (Deutsche Terminbörse) and the floor trading of the LIFFE (London International Financial Futures Exchange). They find that the floor trading turns out to be more attractive in periods of high information intensity, high volatility, high volume, and high trading frequency. The reason they offer for this finding is that market participants infer more information from observing actual trades. Coval and Shumway (2001) confirm the claim that market participants gather all possible information rather than only relying on easily observable data, say, past prices, in determining their trade values. They suggest that subtle but non-transaction signals play important roles. The electronic exchanges they find lose information that can be mirrored by a face-to-face exchange setting. Based on the co-existence of the floor and electronic trading system in the German stock market, Theissen (2002) argues that when employing intra-daily data, both systems contribute to the price discovery process almost equally. Based on testing whether the upstairs intermediation can lower adverse selection cost, Smith et al. (2001) report that the upstairs market is complementary in supplying liquidity. Bessembinder and Venkataraman (2004) show that the upstairs market does a good job of complementing to the electronic exchange because the upstairs market is efficient for many large trades and block-sized trades.

By confirming that market structure does impact the incorporation of news into market prices, Masulis and Shivakumar (2002) compare the speed of adjustment on the NYSE and Nasdaq. They demonstrate that there are faster price adjustments to new information on the Nasdaq. Weston (2002) confirms that the electronic trading system has improved the liquidity of the Nasdaq. Boehmer et al. (2005) suggest that since the electronic trading reveals more information, market quality could be increased by such exposed information in the electronic trading system.

Huang and Stoll (2001) point out that tick size, bid/ask spread, and market depth are not independent from market structure. They are linked to market structure. It is necessary to take account of tick size, bid/ask spread, and market depth when analyzing the market structure. Numerous studies investigate how market structure impacts price discovery and trading costs in different securities markets. Chung and Van Ness (2001) show that after introducing new order handling rules in the Nasdaq, bid/ask spreads decreased, confirming that market structure has a significant effect on trading costs and the price forming process.

Market transparency is the ability of market participants to observe information about the trading process, see O'Hara (1995). Referring to the time of the trade, Harris (2003) defines ex ante (pre-trade) transparency and ex post (post trade) transparency. Comparing market transparency is complicated by the lack of absence of a criterion that can be used to judge the superiority of one trading system over another such as floor market versus electronic market, anonymous trading versus disclosure trading, and auction system versus dealer system. According to Madhavan (2000), there is broad agreement of the influence of market transparency. Market transparency does affect informative order flow and the process of price discovery. Madhavan also points out that while partial disclosure will improve liquidity and reduce trading costs, complete disclosure will reduce liquidity, a situation Harris (2003) refers to as 
the "ambivalence" from the viewpoint of trader's psychology. By investigating the OpenBook ${ }^{1}$ in NYSE, Boehmer et al. (2005) find there is a higher cancel rate and a short time-to-cancel of limit orders in the book, suggesting that traders attempt to manipulate the exposure of their trades. By confirming market design does impact the trading strategy of investors, they support increasing pre-trade transparency and suggest that market quality can be enhanced by greater transparency of the limit order book.

\subsection{Studies of Trade Duration}

There is considerable interest in the information content and implications of the spacing between consecutive financial transactions (referred to as trade duration) for trading strategies and intra-day risk management. Market microstructure theory, supported by empirical evidence, suggests that the spacing between trades be treated as a variable to be explained or predicted since time carries information and closely correlates with price volatility (see, Bauwens and Veredas (2004), Engle (2000), Engle and Russell (1998), Hasbrouck (1996), and O'Hara (1995)). Manganelli (2005) finds that returns and volatility directly interact with trade durations and trade order size. Trade durations tend to exhibit long-range dependence, heavy tailedness, and clustering (see, Bauwens and Giot (2000), Dufour and Engle (2000), Engle and Russell (1998), and Jasiak (1998)). Several studies have modeled durations based on point processes in order to discover the information they contain. Before reviewing the major studies of trade duration in this section, we will introduce the mathematics underlying duration models, that is, point processes.

\subsubsection{Point Processes in Modeling Duration}

Point processes are stochastic processes. Let us first define the stochastic process. Given a probability space $(\Omega, \mathcal{A}, \mathrm{P})$, a family of random variables $\left(X_{t}\right)_{t \in \mathcal{T}}$ on $\Omega$ with values in some set $M$, (i.e., for all $t \in \mathcal{T}$ and $\mathcal{T}$ is some index set), $X_{t}:(\Omega, \mathcal{A}) \rightarrow(\mathrm{M}, \mathcal{B})$ is defined as a stochastic process with index set $\mathcal{T}$ and state space $M$. The point process is then the sequence $\left(T_{n}\right)_{n \in N}$ of positive real random variables if $T_{n}(\omega)<T_{n+1}(\omega)$ for all $\omega \in \Omega$ and all $n \in N$; and $\lim _{n \rightarrow \infty} T_{n}(\omega)=\infty$, for all $\omega \in \Omega$. In this definition, $T_{n}$ is called the $n$th arrival time and $T_{n}=\sum_{i=1}^{n} \tau_{i}$; and $\tau_{n}=T_{n}-T_{n-1}$ (where $\tau_{1}=T_{1}$ ) is called the $n$th waiting time (duration) for the point process.

Point processes and counting processes are tightly connected. A stochastic process $\left(N_{t}\right)_{t \in[0, \infty)}$ is a counting process if: $N_{t}:(\Omega, \mathcal{A}) \rightarrow\left(\mathrm{N}_{0}, \mathrm{P}\left(\mathrm{N}_{0}\right)\right)$ for all $t \geq 0, N_{0} \equiv 0 ; N_{s}(\omega) \leq N_{t}(\omega)$, for all $0 \leq s<t$ and all $\omega \in \Omega ; \lim _{s \rightarrow t, s>t} N_{s}(\omega)=N_{t}(\omega)$, for all $t \geq 0$ and all $\omega \in \Omega ; N_{t}(\omega)-\lim _{s \rightarrow t, s>t} N_{s}(\omega) \in(0,1)$, for all $t>0$ and all $\omega \in \Omega$; and $\lim _{t \rightarrow \infty} N_{t}(\omega)=\infty$, for all $\omega \in \Omega$. A point process $\left(T_{n}\right)_{n \in N}$ corresponds to a counting process $\left(N_{t}\right)_{t \in[0, \infty)}$ and vice versa, i.e.,

$$
N_{t}(\omega)=\left|\left\{n \in N: T_{n}(\omega) \leq t\right\}\right|
$$

for all $\omega \in \Omega$ and all $t \geq 0$. For all $\omega \in \Omega$ and all $n \in N$,

$$
T_{n}(\omega)=\min \left\{t \geq 0: N_{t}(\omega)=n\right\}
$$

The mean value function of the counting process is $m(t)=E\left(N_{t}\right)$, for $t \geq 0$, and $m:[0, \infty) \rightarrow$ $[0, \infty), m(0)=0 . m$ is an increasing and a right continuous function with $\lim _{t \rightarrow \infty} m(t)=\infty$. If the

\footnotetext{
${ }^{1}$ OpenBook was introduced in January 2002 allowing traders off the NYSE floor exchange to find each price in real time for all listed securities. Before OpenBook, only best bid/ask could be observed.
} 
mean value function is differentiable at $t>0$, then the first-order derivative is called the intensity of the counting process. Defining $\lambda(t)=\mathrm{d} m(t) / \mathrm{d} t$,

$$
\lim _{\Delta t \rightarrow 0, \Delta t \neq 0} \frac{1}{|\Delta t|} P\left(\left|N_{t+\Delta t}-N_{t}\right|=1\right)=\lambda(t)
$$

and

$$
\lim _{\Delta t \rightarrow 0, \Delta t \neq 0} \frac{1}{|\Delta t|} P\left(\left|N_{t+\Delta t}-N_{t}\right| \geq 2\right)=0
$$

From the viewpoint of the point processes intra-daily financial data can be described as marked point processes; that is, the state space $M$ is a product space of $R^{2} \otimes \mathcal{M}$ where $\mathcal{M}$ is the mark space. Engle (2000) pointed out that ultra-high frequency transaction data contain two types of processes: (1) time of transactions and (2) events observed at the time of the transaction. Those events can be identified or described by marks, such as trade prices, posted bid and ask price, and volume. As explained earlier, the amount of time between events is the duration. The intensity is used to characterize the point processes and is defined as the expected number of events per time increment considered as a function of time. In survival analysis, the intensity equals the hazard rate. For $n$ durations, $d_{1}, d_{2}, \ldots, d_{n}$, which are sampled from a population with density function $f$ and corresponding cumulative distribution function $F$, the survival function $S(t)$ is:

$$
S(t)=P\left[d_{i}>t\right]=1-F(t)
$$

and the intensity or hazard rate $\lambda(t)$ is:

$$
\lambda(t)=\lim _{\Delta t \rightarrow 0} \frac{P\left[t<d_{i} \leq t+\Delta t \mid d_{i}>t\right]}{\Delta t}
$$

The survival function and the density function can be obtained from the intensity,

$$
\lambda(t)=\frac{f(t)}{S(t)}=\frac{-\mathrm{d} \log (S(t))}{\mathrm{d} t}
$$

\subsubsection{Major Duration Models}

Several models have been proposed to model durations by estimating the intensity. The favored models in the literature is the autoregressive conditional duration (ACD) model proposed by Engle and Russell (1998), the stochastic conditional duration (SCD) model by Bauwens and Veredas (2004), and the stochastic volatility duration (SVD) model by Ghysels et al. (2004).

The ACD model expresses the conditional expectation of duration as a linear function of past durations and past conditional expectation. The disturbance is specified as an exponential distribution and as an extension the Weibull distribution. The SCD model assumes that a latent variable drives the movement of durations. Then expected durations in the SCD model is expressed by observed durations driven by a latent variable. The SVD model seeks to capture the mean and variance of durations.

After these models were proposed, the following extensions appeared in the literature:

- Jasiak (1998): the fractional integrated ACD model;

- Bauwens and Giot (2000): the logarithmic ACD model;

- Zhang et al. (2001): the threshold ACD model; 
- Bauwens and Giot (2003): the asymmetric ACD model;

- Feng et al. (2004): the linear non-Gaussian state-space SCD model.

The $\operatorname{ACD}(m, n)$ model specified in Engle and Russell (1998) is

$$
\begin{gathered}
d_{i}=\psi_{i} \varepsilon_{i} \\
\psi_{i}=\omega+\sum_{j=0}^{m} \alpha_{j} d_{i-j}+\sum_{j=0}^{n} \beta_{j} \psi_{i-j}
\end{gathered}
$$

Bauwens and Giot (2000) give the logarithmic version of the ACD model as follows

$$
d_{i}=e^{\psi_{i}} \varepsilon_{i}
$$

Two possible specifications of conditional durations are

$$
\psi_{i}=\omega+\sum_{j=0}^{m} \alpha_{j} \log d_{i-j}+\sum_{j=0}^{n} \beta_{j} \psi_{i-j}
$$

and

$$
\psi_{i}=\omega+\sum_{j=0}^{m} \alpha_{j} \log \varepsilon_{i-j}+\sum_{j=0}^{n} \beta_{j} \psi_{i-j}
$$

Zhang et al. (2001) extend the conditional duration to a switching-regime version. Defining $L_{q}=$ $\left[l_{q-1}, l_{q}\right)$, and $q=1,2, \ldots, Q$ for a positive integer $Q$, where $-\infty=l_{0}<l_{1}<\ldots<l_{q}=+\infty$ are the threshold values, $d_{i}$ follows a $q$-regime threshold $\operatorname{ACD}(\operatorname{TACD}(m, n))$ model; that is:

$$
\psi_{i}=\omega^{(q)}+\sum_{j=0}^{m} \alpha_{j}^{(q)} d_{i-j}+\sum_{j=0}^{n} \beta_{j}^{(q)} \psi_{i-j}
$$

For example, if there is a threshold value $l_{h}$ and $0<h<q$, the TACD(1,1) model can be expressed as follows:

$$
\psi_{i}=\left\{\begin{array}{lll}
\omega_{1}+\alpha_{1} d_{i-1}+\beta_{1} \psi_{i-1} & \text { if } & 0<d_{i-1} \leq l_{h} \\
\omega_{2}+\alpha_{2} d_{i-1}+\beta_{2} \psi_{i-1} & \text { if } & l_{h}<d_{i-1}<\infty
\end{array}\right.
$$

The threshold $l_{h}$ determines the regime boundaries.

Fernandes and Gramming (2005) propose nonparametric tests for ACD models and suggested the practical application for estimation of intra-daily volatility patterns. Sun et al. (2006) model the trade durations of 18 Dow Johns index component stocks using the ACD model with the positive defined fractal processes and find that these fractal processes do describe the data better than the ACD model with i.i.d. distributions.

The SCD model given by Bauwens and Veredas (2004) takes the following form:

$$
d_{i}=\Psi_{i} \varepsilon_{i}
$$

where

$$
\Psi_{i}=e^{\psi_{i}}
$$




$$
\psi_{i}=\omega+\beta \psi_{i-1}+u_{i}
$$

in which $|\beta|<1$, denotes $I_{i-1}$ the information set before $d_{i}, u_{i}\left|I_{i-1} \sim N\left(0, \sigma^{2}\right), \varepsilon_{i}\right| I_{i-1}$ follows some distribution with positive support, and $u_{i}$ is independent of $\varepsilon_{j} \mid I_{i-1}$ for any $i$ and $j$.

Ghysels et al. (2004) propose a SVD model by assuming that durations are independently and exponentially distributed with gamma heterogeneity. More explicitly, their model can be expressed as:

$$
d_{i}=\frac{U_{i}}{c V_{i}}
$$

where $U_{i}$ and $V_{i}$ are two independent variables with exponential distribution and gamma( $\left.a, a\right)$ distribution. Then this expression can be transferred with suitable nonlinear transformations to the expression with Gaussian factors:

$$
d_{i}=\frac{\Psi\left(1, \Phi\left(F_{1}\right)\right)}{c \Psi\left(a, \Phi\left(F_{2}\right)\right)}=\frac{H\left(1, F_{1}\right)}{c H\left(1, F_{2}\right)}
$$

where $F_{1}$ and $F_{2}$ are i.i.d standard normal variables, $\Phi$ is the cdf of the standard normal distribution, and $\Psi(a,$.$) is the quantile function of the gamma (a, a)$ distribution.

\section{Long-Range Dependence}

Long-range dependence is the dependence structure across long time periods. As stated earlier, it denotes the property of a time series to exhibit persistent behavior, i.e., a significant dependence between very distant observations and a pole in the neighborhood of the zero frequency of their spectrum. In time domain, if $\left\{X_{t}, t \in T\right\}$ exhibits long-range dependence, its autocovariance function $\gamma(k)$ has the property of $\sum|\gamma(k)|=\infty$, where $k$ measures the distance between two observations, i.e., the order of lags. In frequency domain, if $\left\{X_{t}, t \in T\right\}$ exhibits long-range dependence, its spectral density $f(\lambda)$ $(-\pi<\lambda<\pi)$ has a "pole" at frequency zero, i.e., $f(0)=1 / 2 \pi \sum_{k=-\infty}^{\infty} \gamma(k)=\infty$.

\subsection{Estimation and Detection of LRD in Time Domain}

\subsubsection{The Rescaled Adjusted Range Approach}

The rescaled adjusted range method, denoted by $R / S$, was proposed by Hurst (1951) and discussed in detail in Mandelbrot and Wallis (1969), Mandelbrot (1975), Mandelbrot and Taqqu (1979), and Beran (1994). For a time series, $\left\{X_{t}, t \geq 1\right\}$, let $Y_{T}=\sum_{t=1}^{T} X_{t}$ and

$$
S^{2}(t, k)=\frac{1}{k} \sum_{i=t+1}^{t+k}\left(X_{i}-\bar{X}_{t, k}\right)^{2}
$$

where $\bar{X}_{t, k}=k^{-1} \sum_{i=t+1}^{t+k} X_{i}$, then define the adjusted range

$$
R(t, k)=\max _{0 \leq i \leq k}\left[Y_{t+i}-Y_{t}-\frac{i}{k}\left(Y_{t+k}-Y_{t}\right)\right]-\min _{0 \leq i \leq k}\left[Y_{t+i}-Y_{t}-\frac{i}{k}\left(Y_{t+k}-Y_{t}\right)\right]
$$

the standardized ratio $R(t, k) / S(t, k)$ is the rescaled adjusted range, i.e., the $\mathrm{R} / \mathrm{S}$ statistic. Hurst observed that for a large $k$ based on the Nile River data, $\log E[R / S] \approx a+H \log k$ with $H>0.5$. To determine $H$ by using the $R / S$ statistic, we can do the following:

1. Divide the time series of length $N$ into $K$ blocks. 
2. For each lag $t$, starting at points $t_{i}=i T / K+1$, compute $R\left(k_{i}, t\right) / S\left(k_{i}, t\right), i=1,2, \cdots$, for all possible $k$ such that $t_{i}+k \leq N$.

3. Plot its logarithm against the logarithm of $k$. This plot is sometimes called the pox plot for the $R / S$ statistic.

4. The parameter $H$ is the estimated slope of the line in the pox plot.

The $R / S$ method requires cutting off both the low and high end of the plot to make reliable estimates. The low end of the plot stands for the short-range dependence in the time series and there are too few points on the high end. In the literature it is also argued that the $R / S$ cannot provide the confidence intervals for the estimates and cannot discriminate slight LRD from no LRD. Compared with other methods, the $R / S$ approach is less efficient. When the time series is non-stationary and departs from the normal distribution, this method is not robust, see Lo (1991), Taqqu et al. (1995), and Taqqu and Teverovsky (1998).

Lo modifies the $R / S$ approach and proposes a test procedure for the null hypothesis of no LRD. In Lo's method, he suggests using a weighted sum of autocovariance for $S$ instead of the sample standard deviation to normalize $R$. Meantime, his modification suggests not considering multiple lags but only using the length $N$ of the series, i.e.,

$$
S_{q}^{2}(N)=\frac{1}{N} \sum_{j=1}^{N}\left(X_{j}-\bar{X}_{N}\right)^{2}+\frac{2}{N} \sum_{j=1}^{q} \omega_{j}(q)\left(\sum_{i=j+1}^{N}\left(X_{i}-\bar{X}_{N}\right)\left(X_{i-j}-\bar{X}_{N}\right)\right)
$$

where $\bar{X}_{N}$ denotes the sample mean of the time series, and $\omega_{j}(q):=1-\frac{j}{q+1}, q<N$. We can use the following term to represent $S_{q}(N)$ by adding the weighted sample autocovariances to the sample variance, i.e.,

$$
S_{q}^{2}(N)=S^{2}+2 \sum_{j=1}^{q} \omega_{j}(q) \hat{\gamma}_{j}
$$

where $\hat{\gamma}_{j}$ are the sample autocovariances. Lo shows that the distribution of the statistic

$$
V_{q}(N):=\frac{N^{-1 / 2} R(N)}{S_{q}(N)}
$$

is asymptotic to

$$
W_{1}=\max _{0 \leq t \leq 1} W_{0}(t)-\min _{0 \leq t \leq 1} W_{0}(t)
$$

where $W_{0}$ is the standard Brownian bridge. This fact allows the computation of a $95 \%$ confidence interval for $W_{1}$. Thus, Lo uses the interval $[0.809,1.862]$ as the asymptotic $95 \%$ acceptance region of the null hypothesis of no LRD.

Since Lo only provides the method to test if LRD is present or not without suggesting an estimator of $H$, Teverovsky et al. (1999) modified Lo's method to get an estimator of $H$. They suggest using $V_{q}$ with a wide range of values of $q$, and then ploting the estimates as was done for the pox plot. 


\subsubsection{ARFIMA Model}

Conventional analysis of time series under the stationary assumption typically relies on the standard integrated autoregressive moving average model, i.e. ARIMA model of following form:

$$
\alpha(L)(1-L)^{d} X_{t}=\beta(L) \varepsilon_{t}
$$

where, $\varepsilon_{t} \sim\left(0, \sigma^{2}\right)$, and $\alpha(L)$ is the autoregressive polynomials in the lag operator $L$ such that $\alpha(L)=$ $1-\alpha_{1}(L)-, \ldots,-\alpha_{p}(L)^{p}$. $\beta(L)$ is the moving average polynomials in the lag operator $L$ such that $\beta(L)=1+\beta_{1}(L)-, \ldots,-\beta_{q}(L)^{q}$. All roots of $\alpha(L)$ and $\beta(L)$ lie outside the unit circle. Granger and Joyeux (1980) and Hosking (1981) generalize $d$ to a non-integer value by the fractional differencing operator defined by

$$
(1-L)^{d}=\sum_{k=0}^{\infty} \frac{\Gamma(k-d) L^{k}}{\Gamma(-d) \Gamma(k+1)}
$$

where $\Gamma(\cdot)$ is gamma function. That is the Autoregressive Fractional Integrated Moving Average (ARFIMA) model allows a non-integer value of $d$. The ARFIMA model has the ability to capture significant dependence between distant observations compared with ARIMA model. Hosking (1981) shows that autocorrelation function $\rho(k)$ of an ARFIMA process has a slower hyperbolic decay pattern, that is $\rho(k) \sim k^{2 d-1}$, with $d<0.5$ when $k \rightarrow \infty$ and the autocorrelation of ARIMA decay follows an exponential pattern, that is, $\rho(k) \sim r^{k}$, with $r \in(0,1)$ when $k \rightarrow \infty$. The memory of time series is captured by $d$, therefore the existence of long memory can be tested based on the statistical significance of the fractional differencing parameter $d$.

For the first different of the series $Y_{t}, Y_{t}=(1-L) X_{t}$, the equation

$$
(1-L)^{d} X_{t}=\alpha^{-1}(L) \beta(L) \varepsilon_{t}=u_{t}
$$

can be used to estimate $d$. In this case, the Hurst index is $1 / 2+d$.

\subsubsection{Variance-Type Method}

Teverovsky and Taqqu (1995), Taqqu et al. (1995), and Taqqu and Teverovsky (1996) discuss the variance-type methods for estimating the Hurst index: the aggregated variance method and differenced variance method.

For the aggregated variance method, the variance of $X$ is of order $N^{2 H-2}$ suggesting:

1. For an integer $m$ between 2 and $N / 2$, divide a given series of length $N$ into blocks of length $m$, and compute the sample mean over each $k$-th block.

$$
\bar{X}_{k}^{(m)}:=\frac{1}{m} \sum_{t=(k-1) m+1}^{k m} X_{t}
$$

where $k=1,2, \cdot,[N / m]$

2. For each $m$, compute the sample variance of $\bar{X}_{k}^{(m)}$,

$$
\bar{s}_{m}^{2}:=\frac{1}{[N / m]-1} \sum_{k=1}^{[N / m]}\left(\bar{X}_{k}^{(m)}-\bar{X}\right)^{2}
$$


3. Plot $\log s_{m}^{2}$ against $\log m$

4. For the large values of $m$, the result should be a straight line with a slope of $2 H-2$. Then the slope can be estimated by fitting a least-squares line in the log-log plot. If the series has no long-range dependence and finite variance, then $H=0.5$ and the slope of the fitted line is -1 .

There are two types of non-stationarity, one is jumps in the mean and the other is slowly declining trends. Teverovsky and Taqqu (1995) distinguish these from long-range dependence by using the differenced variance method. They difference the variance and study the sample variance $\bar{s}_{m_{i}+1}^{2}-\bar{s}_{m_{i}}^{2}$, where $m_{i}$ are the successive values of $m$ as defined above. Using it together with the original aggregated variance method, the difference variance method can detect the presence of the two types of non-stationary effects mentioned above.

Another method involving the variance is the variance of residuals method introduced by Peng et al. (1994). Similar to the aggregated variance method, the series is divided into blocks with size of $m$. Next, the partial sums are computed within each block, i.e.,

$$
Y(k)^{(m)}:=\sum_{t=(k-1) m+1}^{k m} X_{t}
$$

where $k=1,2, \cdots,[N / m]$. A regression line $a+b k$ is fitted to the partial sums within each block, and the sample variance of the residuals $\bar{s}^{(m)}$ is computed. Taqqu et al. (1995) prove that in the Gaussian case, the variance of residuals is proportional to $m^{2 H}$. By plotting $\log \bar{s}^{(m)}$ against $\log m$, the slope, i.e., $2 H$, can be estimated (see Taqqu and Teverovsky (1996) for more details).

\subsubsection{Absolute Moments Method}

The absolute moments method is a generalization of the aggregated variance method. Using this method

1. For an integer $m$ between 2 and $N / 2$, divide a given series of length $N$ into blocks of length $m$, and compute the sample mean over each $k$-th block.

$$
\bar{X}_{k}^{(m)}:=\frac{1}{m} \sum_{t=(k-1) m+1}^{k m} X_{t}
$$

where $k=1,2, \cdot,[N / m]$

2. For each $m$, compute the $n$-th absolute moment of $\bar{X}_{k}^{(m)}$,

$$
A M_{n}^{(m)}=\frac{1}{[N / m]-1} \sum_{k=1}^{[N / m]}\left|\bar{X}_{k}^{(m)}-\bar{X}\right|^{n}
$$

3. The $A M_{n}^{(m)}$ is asymptotically proportional to $m^{n(H-1)}$.

4. Plot $\log A M_{n}^{(m)}$ against $\log m$.

5. For the large values of $m$, the result should be a straight line with a slope of $n(H-1)$. Then the slope can be estimated by fitting a least-squares line in the log-log plot. If the series has no long-range dependence and finite variance, then $H=0.5$ and the slope of the fitted line is $-n / 2$. 
Similar to the absolute moments method, Higuchi (1988) suggests the fractal dimension method, see Taqqu and Teverovsky (1998). The difference between these two methods is that the absolute moments method (when $n=1$ ) uses a moving window to compute the aggregated series, while the fractal dimension method uses the non-intersecting blocks. The fractal dimension method requires intensive computation and increases accuracy in shorter time series. Taqqu et al. (1995), and Taqqu and Teverovsky (1998) discuss these two methods in detail.

\subsection{Estimation and Detection of LRD in Frequency Domain}

\subsubsection{Periodogram Method}

Geweke and Porter-Hudak (1983) introduced a semi-nonparametric procedure to test long memory based on the slope of spectral density around the angular frequency $\omega=0$. For the periodogram of $X_{t}$ at frequency $\omega_{j}$, i.e., $g(\omega)$, which is defined as follows

$$
g(\omega)=\frac{1}{2 \pi T}\left|\sum_{t=1}^{T} e^{i t \omega}\left(X_{t}-\bar{X}\right)\right|^{2}
$$

the differencing parameter $d$ can be consistently estimated by the regression

$$
\ln g(\omega)=c-d \ln \left(4 \sin ^{2}\left(\frac{\omega_{j}}{2}\right)\right)+\eta_{j}, \quad j=1,2, \ldots, n
$$

where $\omega_{j}=2 \pi j / T,(j=1, \ldots, T-1)$ denotes the Fourier frequencies of the sample, $T$ is the sample

size, and $n=f(T)<<T$ is the number of Fourier frequencies included in the spectral regression. As Geweke and Porter-Hudak (1983) show, the slope of the line in log-log plot is $1-2 H$.

Extensions and improvements to the periodogram method, for example, the continuous periodogram method and the averaged (comulative) periodogram method, have been discussed in the literature, see, for example, Robinson (1995a), Taqqu et al. (1995), Taqqu and Teverovsky (1998), Moulines and Soulier (1999), and Hurvich and Brodski (2001).

\subsubsection{Whittle-Type Methods}

The Whittle estimator is the extension of the periodogram method. If the time series $X_{t}$ follows Gaussianity, the Gaussian maximum likelihood estimate (MLE) might have optimal asymptotic statistical properties and can be used for approximation, see Whittle (1951) and Hannan (1973). The periodogram of $X_{t}$ at frequency $\omega_{j}$ is defined as $g(\omega)$,

$$
g(\omega)=\frac{1}{2 \pi T}\left|\sum_{t=1}^{T} X_{t} e^{i t \omega}\right|^{2}
$$

which is an estimator of the spectral density. It is evaluated at the Fourier Frequencies $\omega_{j}=2 \pi j T$.

Beran (1994) shows that the following equation is an approximation to the Gaussian likelihood,

$$
L_{W}(\theta)=-\frac{1}{2 \pi} \sum_{j=1}^{[T / 2]} \log f_{\theta}\left(\omega_{j}\right)+\frac{I_{N}\left(\omega_{j}\right)}{f_{\theta}\left(\omega_{j}\right)}
$$


and the Whittle estimator is found by minimizing it for a given parametric spectral density $f_{\theta}(\omega)$. The Gaussian likelihood can be replaced by different approximations without affecting first-order limit distributional characteristics. Robinson (2003) shows that the estimates which maximize such approximations are all $\sqrt{n}$-consistent and of the same limit normal distribution as the Gaussian MLE. Fox

and Taqqu (1986) show the Whittle estimate $\hat{H}$ of $H$ is asymptotically normal with rate of convergence $T^{1 / 2}$ and the asymptotic distribution of $\sqrt{T}(\hat{H}-H)$ is Gaussian. Relaxing Gaussianity, Giraitis and Surgailis (1990) discuss the properties of the Whittle estimate $\hat{H}$ of $H$.

Robinson (1995b) develops the local Whittle method and Taqqu and Teverovsky (1998) provide further discussion about it. The local Whittle method is a semi-parametric estimator. It only specifies the parametric form of the spectral density with $\omega$ approaching to zero. It assumes that

$$
f_{c, H}(\omega)=c \omega^{1-2 H}
$$

for frequencies $\omega$ close to the origin. One estimate minimizes

$$
\sum_{j=1}^{m} \log f_{c, H}\left(\omega_{j}\right)+\frac{I_{T}\left(\omega_{j}\right)}{f_{c, H}\left(\omega_{j}\right)}
$$

with respect to $c$ and $H$ for some $m<[T / 2], T$ being the length of the data.

Taqqu and Teverovsky (1998) introduce the aggregated Whittle method which provides a robust Whittle estimator without considering exact parametric information about the spectral density. It can be used for longer time series. This method suggests aggregating the data to create a shorter series.

$$
X_{i}:=\frac{1}{m} \sum_{t=m(i-1)+1}^{m t} X_{t}
$$

If the aggregation level of $m$ is high enough and long-range dependence occurs, then the new series will approach to a fractional Gaussian noise. In the finite variance case, the Whittle estimator can increase the estimation accuracy with an underlying fractional Gaussian noise assumption.

\subsection{Econometric Modeling of LRD}

Several econometric models have been extended to describe long-range dependence, for example, extending the ARMA model to the ARFIMA model discussed in the previous section. In this section, we introduce four types of extensions, i.e., GARCH-type extension, stochastic volatility type extension, unit root type extension, and regime switching type extension.

\subsubsection{GARCH-Type Extension}

Robinson (1991) suggests extending GARCH model by using fractional differences in order to accommodate the existence of long-range dependence. The fractional differencing operator is defined as in equation (43) by Baillie et al. (1996). The fractionally integrated GARCH (FIGARCH) model is then

$$
(1-L)^{d} \beta(L)\left(h_{t}^{2}-\mu\right)=\alpha(L)\left(r_{t}^{2}-\mu\right)
$$


For the well-defined process, the parameters $\alpha_{j}, \beta_{j}$, and $d$ are constrained. Then the coefficients $\theta_{j}$ are all nonnegative in

$$
h_{t}^{2}=\mu+(1-L)^{-d} \alpha(L) \beta^{-1}(L) r_{t}^{2}=\mu+\sum_{j=0}^{\infty} \theta\left(r_{t-1-j}^{2}-\mu\right)
$$

Implied by equation (49), the parameters $\alpha_{j}$ and $\beta_{j}$ are constrained as in the standard GARCH model. This also implies that the parameter $d$ is constrained to be positive. Breidt et al. (1998) argue that the $r_{t}$ in equation (49) is not covariance stationary and the autocovariance function of $r_{t}$ is not defined. Bollerslev and Mikkelsen (1996) formulate a fractionally integrated EGARCH model of the following form

$$
\log h_{t}^{2}=\mu_{t}+\theta(L) \phi(L)^{-1}(1-L)^{-d} g\left(\epsilon_{t-1}\right)
$$

where $\theta(z)=1+\theta_{1} z+, \cdots,+\theta_{p} z^{p}$ for $|z| \leq 1$ is an autoregressive polynomial, and $\phi(z)=1-$ $\phi_{1} z-, \cdots,-\phi_{p} z^{p}$ is a moving average polynomial and $\phi(z)$ has no roots in common with $\theta(z)$. The fractional integrated EGARCH model gives a strictly stationary and ergodic process. Nelson (1991) shows that $\left(\log h_{t}^{2}-\mu_{t}\right)$ is covariance stationary under certain condition and $d<0.5$.

\subsubsection{Stochastic Volatility Type Extension}

A long-range dependence stochastic volatility model is discussed by Breidt et al. (1998). The stochastic volatility model is defined by

$$
r_{t}=h_{t} \epsilon_{t}, \quad h_{t}=h \exp \left(u_{t} / 2\right)
$$

where $u_{t}$ is independent of $\epsilon_{t}, \epsilon_{t}$ is independent and identically distributed (i.i.d.) with mean zero and variance one. $u_{t}$ in a simple long-range dependence model can be defined as

$$
(1-L)^{d} u_{t}=\eta_{t}
$$

where $\eta_{t}$ follows i.i.d. normal distribution with zero mean and variance $\sigma_{\eta}^{2}$, and $d \in(-0.5,0.5)$. For long-range dependence, $u_{t}$ can be expressed as an ARFIMA $(p, d, q)$ process, defined as

$$
(1-L)^{d} \phi(L) u_{t}=\theta(L) \eta_{t}
$$

where $\eta_{t}$ follows i.i.d. normal distribution with zero mean and variance $\sigma_{\eta}^{2}$.

\subsubsection{Unit Root Type Extension}

Robinson (1994) considers following model that nests a unit root model in order to grasp the effect of long-range dependence:

$$
\begin{gathered}
\phi(L) r_{t}=\epsilon_{t}, \quad t \geq 1 \\
r_{t}=0, \quad t \leq 0
\end{gathered}
$$

where $\epsilon_{t}$ is an $I(0)$ process with parametric autocorrelation and

$$
\phi(L)=(1-L)^{d_{1}}(1+L)^{d_{2}} \prod_{j=3}^{n}\left(1-2 \cos \omega_{j} L+L^{2}\right)^{d_{j}}
$$


where $\omega_{j}$ are given distinct real numbers in $(0, \pi)$, and the $d_{j}, 0 \leq j \leq n$, are arbitrary real numbers. This model also covers seasonal and cyclical components. Velasco and Robinson (2000) propose the following model

$$
\begin{gathered}
(1-L)^{s} r_{t}=\epsilon_{t}, \quad t \geq 1, \\
r_{t}=0, \quad t \leq 0, \\
(1-L)^{d-s} \epsilon_{t}=u_{t}, \quad t=0, \pm 1, \cdots,
\end{gathered}
$$

where $s$ is the integer part of $d+1 / 2$ and $u_{t}$ is a parametric $I(0)$ process. $\epsilon_{t}$ is a stationary $I(d-s)$ process. Marinucci and Robinson (1999) discuss the difference between the two models given by equations (51)(52) and equations (54)-(56) with respect to the two definitions of nonstationarity $I(d)$ processes.

\subsubsection{Regime Switching Type Extension}

Diebold and Inoue (2001) show that long-range dependence models and regime switching models are intimately related in several circumstances, including a simple mixture model, stochastic permanent break model, and Markov-switching model. They demonstrate that with suitably adapted time varying transition probabilities these regime switching models can generate an autocovariance structure. This autocovariance structure is similar to the fractionally integrated processes. Banerjee and Urga (2005) provide an overiew of the recent development in the studies of modeling regime switching and long-range dependence.

Haldrup and Nielsen (2006) propose a regime switching multiplicative seasonal ARFIMA model that accommodates both fractional integration and regime switching simultaneously. The model is:

$$
A_{s_{t}}(L)\left(1-\alpha_{s_{t}} L^{24}\right)(1-L)^{d_{s_{t}}}\left(y_{t}-\mu_{s_{t}}\right)=\epsilon_{s_{t}, t}, \quad \epsilon_{s_{t}, t} \sim \operatorname{nid}\left(0, \sigma_{s_{t}}^{2}\right)
$$

where $A_{s_{t}}(L)$ is an eighth order lag polynomial capturing the within-the-day effects. The polynomial $\left(1-\alpha_{s_{t}} L^{24}\right)$ stands for a daily quasi-difference filter, $s_{t}=0,1$ denotes the regime determined by a Markov chain with transition probabilities, and $\mu$ is the mean.

\section{Fractal Processes and Long-Range Dependence}

Fractal processes (self-similar processes) are tightly connected with the analysis of long-range dependence. Self-similar processes are invariant in distribution with respect to changes of time and space scale. The scaling coefficient or self-similarity index is a non-negative number denoted by $H$, the Hurst parameter. If $\{X(t+h)-X(h), t \in T\} \stackrel{d}{=}\{X(t)-X(0), t \in T\}$ for all $h \in T$, the real-valued process $\{X(t), t \in T\}$ has stationary increments. Samorodnisky and Taqqu (1994) provide a succinct expression of self-similarity: $\{X(a t), t \in T\} \stackrel{d}{=}\left\{a^{H} X(t), t \in T\right\}$. The process $\{X(t), t \in T\}$ is called $H$-sssi if it is self-similar with index $H$ and has stationary increments. Long-range dependence processes are asymptotically second-order self-similar (see, Willinger et al (1998)). 


\subsection{Specification of the Fractal Processes}

Lamperti (1962) first introduced semi-stable processes (which we nowadays call self-similar processes). Let $T$ be either $R, R_{+}=\{t: t \geq 0\}$ or $\{t: t>0\}$. Then the real-valued process $\{X(t), t \in T\}$ is self-similar with Hurst index $H>0(H$-ss $)$ for any $a>0$ and $d \geq 1, t_{1}, t_{2}, \ldots, t_{d} \in T$, satisfying:

$$
\left(X\left(a t_{1}\right), X\left(a t_{2}\right), \ldots, X\left(a t_{d}\right)\right) \stackrel{d}{=}\left(a^{H} X\left(t_{1}\right), a^{H} X\left(t_{2}\right), \ldots a^{H} X\left(t_{d}\right)\right) .
$$

\subsubsection{Fractional Gaussian Noise}

For a given $H \in(0,1)$ there is basically a single Gaussian $H$-sssi process, namely fractional Brownian motion (fBm) that was first introduced by Kolmogorov (1940). Mandelbrot and Wallis (1968) and Taqqu (2003) clarify the definition of fBm as a Gaussian $H$-sssi process $\left\{B_{H}(t)\right\}_{t \in R}$ with $0<H<1$. Mandelbrot and van Ness (1968) defined the stochastic representation

$$
B_{H}(t):=\frac{1}{\Gamma\left(H+\frac{1}{2}\right)}\left(\int_{-\infty}^{0}\left[(t-s)^{H-\frac{1}{2}}-(-s)^{H-\frac{1}{2}}\right] d B(s)+\int_{0}^{t}(t-s)^{H-\frac{1}{2}} d B(s)\right)
$$

where $\Gamma(\cdot)$ represents the Gamma function:

$$
\Gamma(a):=\int_{0}^{\infty} x^{a-1} e^{-x} d x
$$

and $0<H<1$ is the Hurst parameter. The integrator $B$ is the ordinary Brownian motion. The main difference between fractional Brownian motion and ordinary Brownian motion is that the increments in Brownian motion are independent while in fractional Brownian motion they are dependent. As to the fractional Brownian motion, Samorodnitsky and Taqqu (1994) define its increments $\left\{Y_{j}, j \in Z\right\}$ as fractional Gaussian noise (fGn), which is, for $j=0, \pm 1, \pm 2, \ldots, Y_{j}=B_{H}(j-1)-B_{H}(j)$.

\subsubsection{Fractional Stable Noise}

Fractional Brownian motion can capture the effect of long-range dependence, but has less power to capture heavy tailedness. The existence of abrupt discontinuities in financial data, combined with the empirical observation of sample excess kurtosis and unstable variance, confirms the stable Paretian hypothesis first identified by Mandelbrot $(1963,1983)$. It is natural to introduce stable Paretian distribution in self-similar processes in order to capture both long-range dependence and heavy tailedness. Samorodinitsky and Taqqu (1994) introduce the $\alpha$-stable $H$-sssi processes $\{X(t), t \in R\}$ with $0<\alpha<2$. If $0<\alpha<1$, the Hurst parameter values are $H \in(0,1 / \alpha]$ and if $1<\alpha<2$, the Hurst parameter values are $H \in(0,1]$. There are many different extensions of fractional Brownian motion to the stable distribution. The most commonly used is the linear fractional stable motion (also called linear fractional Lévy motion), $\left\{L_{\alpha, H}(a, b ; t), t \in(-\infty, \infty)\right\}$, which is defined by Samorodinitsky and Taqqu (1994) as follows:

$$
L_{\alpha, H}(a, b ; t):=\int_{-\infty}^{\infty} f_{\alpha, H}(a, b ; t, x) M(d x)
$$

where

$$
f_{\alpha, H}(a, b ; t, x):=a\left((t-x)_{+}^{H-\frac{1}{\alpha}}-(-x)_{+}^{H-\frac{1}{\alpha}}\right)+b\left((t-x)_{-}^{H-\frac{1}{\alpha}}-(-x)_{-}^{H-\frac{1}{\alpha}}\right)
$$


and where $a, b$ are real constants, $|a|+|b|>1,0<\alpha<2,0<H<1 H \neq 1 / \alpha$, and $M$ is an $\alpha$ stable random measure on $R$ with Lebesgue control measure and skewness intensity $\beta(x), x \in(-\infty, \infty)$ satisfying: $\beta(\cdot)=0$ if $\alpha=1$. They define linear fractional stable noises expressed by $Y(t)$, and $Y(t)=X_{t}-X_{t-1}$

$$
\begin{aligned}
Y(t) & =L_{\alpha, H}(a, b ; t)-L_{\alpha, H}(a, b ; t-1) \\
& =\int_{R}\left(a\left[(t-x)_{+}^{H-\frac{1}{\alpha}}-(t-1-x)_{+}^{H-\frac{1}{\alpha}}\right]\right. \\
& \left.+b\left[(t-x)_{-}^{H-\frac{1}{\alpha}}-(t-1-x)_{-}^{H-\frac{1}{\alpha}}\right]\right) M(d x)
\end{aligned}
$$

where $L_{\alpha, H}(a, b ; t)$ is a linear fractional stable motion defined by equation (3), and $M$ is a stable random measure with Lebesgue control measure given $0<\alpha<2$. In this paper, if there is no special indication, the fractional stable noise (fsn) is generated from a linear fractional stable motion.

Some properties of these processes have been discussed in Mandelbrot and Van Ness (1968), Maejima (1983), Maejima and Rachev (1987), Manfields et al. (2001), Rachev and Mittnik (2000), Rachev and Samorodnitsky (2001), Samorodnitsky (1994, 1996, 1998), and Samorodinitsky and Taqqu (1994).

\subsection{Estimation of Fractal Processes}

\subsubsection{Estimating the Self-Similarity Parameter in Fractional Gaussian Noise}

Beren (1994) discusses the Whittle estimation (which we discussed earlier) of the self-similarity parameter. For fractional Gaussian noise, $Y_{t}$, let $f(\lambda ; H)$ denote the power spectrum of $Y$ after being normalized to have variance 1 and let $I(\lambda)$ the periodogram of $Y_{t}$, that is

$$
I(\lambda)=\frac{1}{2 \pi N}\left|\sum_{t=1}^{N} Y_{t} e^{i t \lambda}\right|^{2}
$$

The Whittle estimator of $H$ is obtained by finding $\hat{H}$ that minimizes

$$
g(\hat{H})=\int_{-\pi}^{\pi} \frac{I(\lambda)}{f(\lambda ; \hat{H})} d \lambda
$$

\subsubsection{Estimating the Self-Similarity Parameter in FSN}

Stoev et al (2002) proposed the least-squares (LS) estimator of the Hurst index based on the finite impulse response transformation (FIRT) and wavelet transform coefficients of the fractional stable motion. A FIRT is a filter $v=\left(v_{0}, v 1, \ldots, v_{p}\right)$ of real numbers $v_{t} \in \Re, t=1, \ldots, p$, and length $p+1$. It is defined for $X_{t}$ by

$$
T_{n, t}=\sum_{i=0}^{p} v_{i} X_{n(i+t)}
$$

where $n \geq 1$ and $t \in N$. The $T_{n, t}$ are the FIRT coefficients of $X_{t}$, that is, the FIRT coefficients of the fractional stable motion. The indices $n$ and $t$ can be interpreted as "scale" and "location". If $\sum_{i=0}^{p} i^{r} v_{i}=0$, for $r=0, \ldots, q-1$, but $\sum_{i=0}^{p} i^{q} v_{i} \neq 0$, the filter $v_{i}$ can be said to have $q \geq 1$ zero moments. If $\left\{T_{n, t}, n \geq 1, t \in N\right\}$ is the FIRT coefficients of fractional stable motion with the filter $v_{i}$ that have at 
least one zero moment, Stoev et al. prove the following two properties of $T_{n, t}$ : (1) $T_{n, t+h} \stackrel{d}{=} T_{n, t}$, and (2) $T_{n, t} \stackrel{d}{=} n^{H} T_{1, t}$, where $h, t \in N, n \geq 1$. We suppose that $T_{n, t}$ are available for the fixed scales $n_{j}$ $j=1, \ldots, m$ and locations $t=0, \ldots, M_{j}-1$ at the scale $n_{j}$, since only a finite number, say $M_{j}$, of the FIRT coefficients are available at the scale $n_{j}$.

By using these properties, we have

$$
E \log \left|T_{n_{j}, 0}\right|=H \log n_{j}+E \log \left|T_{1,0}\right|
$$

The left-hand side of this equation can be approximated by

$$
Y_{\log }\left(M_{j}\right)=\frac{1}{M_{j}} \sum_{t=0}^{M_{j}-1} \log \left|T_{n_{j}, t}\right|
$$

Then we get

$$
\left(\begin{array}{c}
Y_{\log }\left(M_{1}\right) \\
\vdots \\
Y_{\log }\left(M_{m}\right)
\end{array}\right)=\left(\begin{array}{cc}
\log n_{1} & 1 \\
\vdots & \vdots \\
\log n_{m} & 1
\end{array}\right)\left(\begin{array}{c}
H \\
E \log \left|T_{1,0}\right|
\end{array}\right)+\left(\begin{array}{c}
\sqrt{M}\left(Y_{\log }\left(M_{1}\right)-E \log \left|T_{n_{1}, 0}\right|\right) \\
\vdots \\
\sqrt{M}\left(Y_{\log }\left(M_{m}\right)-E \log \left|T_{n_{m}, 0}\right|\right)
\end{array}\right)
$$

In short, we can express the above equation as follows

$$
Y=X \theta+\frac{1}{\sqrt{M}} \varepsilon
$$

Equation (66) shows that the self-similarity parameter $H$ can be estimated by a standard linear regression of the vector $Y$ against the matrix $X$. Stoev et al explain this procedure.

\subsubsection{Estimating the Parameters of Stable Paretian Distribution}

Stable distribution requires four parameters for complete description: an index of stability $\alpha \in(0,2]$ (also called the tail index), a skewness parameter $\beta \in[-1,1]$, a scale parameter $\gamma>0$, and a location parameter $\zeta \in \Re$. There is unfortunately no closed-form expression for the density function and distribution function of a stable distribution. Rachev and Mittnik (2000) give the definition of the stable distribution: A random variable $X$ is said to have a stable distribution if there are parameters $0<\alpha \leq 2,-1 \leq \beta \leq 1, \gamma \geq 0$ and $\zeta$ real such that its characteristic function has the following form:

$$
E \exp (i \theta X)=\left\{\begin{aligned}
\exp \left\{-\gamma^{\alpha}|\theta|^{\alpha}\left(1-i \beta(\sin \theta) \tan \frac{\pi \alpha}{2}\right)+i \zeta \theta\right\} & \text { if } \quad \alpha \neq 1 \\
\exp \left\{-\gamma|\theta|\left(1+i \beta \frac{2}{\pi}(\sin \theta) \ln |\theta|\right)+i \zeta \theta\right\} & \text { if } \quad \alpha=1
\end{aligned}\right.
$$

and,

$$
\operatorname{sign} \theta=\left\{\begin{aligned}
1 & \text { if } \quad \theta>0 \\
0 & \text { if } \quad \theta=0 \\
-1 & \text { if } \quad \theta<0
\end{aligned}\right.
$$

Stable density is not only support for all of $(-\infty,+\infty)$, but also for a half line. For $0<\alpha<1$ and $\beta=1$ or $\beta=-1$, the stable density is only for a half line.

In order to estimate the parameters of the stable distribution, the maximum likelihood estimator given in Rachev and Mittnik (2000) has been employed. Given $N$ observations, $X=\left(X_{1}, X_{2}, \cdots, X_{N}\right)^{\prime}$ for the positive half line. The log-likelihood function is of the form 


$$
\ln (\alpha, \lambda ; X)=N \ln \lambda+N \ln \alpha+(\alpha-1) \sum_{i=1}^{N} \ln X_{i}-\lambda \sum_{i=1}^{N} X_{i}^{\alpha}
$$

which can be maximized using, for example, a Newton-Raphson algorithm. It follows from the first-order condition,

$$
\lambda=N\left(\sum_{i=1}^{N} X_{i}^{\alpha}\right)^{-1}
$$

that the optimization problem can be reduced to finding the value for $\alpha$ which maximizes the concentrated likelihood

$$
\ln ^{*}(\alpha ; X)=\ln \alpha+\alpha \nu-\ln \left(\sum_{i=1}^{N} X_{i}^{\alpha}\right)
$$

where $\nu=N^{-1} \Sigma_{i=1}^{N} \ln X_{i}$. The information matrix evaluated at the maximum likelihood estimates, denoted by $I(\hat{\alpha}, \hat{\lambda})$, is given by

$$
I(\hat{\alpha}, \hat{\lambda})=\left(\begin{array}{cc}
N \hat{\alpha}^{-2} & \sum_{i=1}^{N} X_{i}^{\hat{\alpha}} \ln X_{i} \\
\sum_{i=1}^{N} X_{i}^{\hat{\alpha}} \ln X_{i} & N \hat{\lambda}^{-2}
\end{array}\right)
$$

It can be shown that, under fairly mild condition, the maximum likelihood estimates $\hat{\alpha}$ and $\hat{\lambda}$ are consistent and have asymptotically a multivariate normal distribution with mean $(\alpha, \lambda)^{\prime}$ (see Rachev and Mittnik (2000)).

Other methods for estimating the parameters of a stable distribution (i.e., the method of moments based on the characteristic function, the regression-type method, and the fast Fourier transform method) are discussed in Stoyanov and Racheva-Iotova (2004a, 2004b, 2004c).

\subsection{Simulation of Fractal Processes}

\subsubsection{Simulation of Fractional Gaussian Noise}

Paxson (1997) provides a method to generate the fractional Gaussian noise by using the Discrete Fourier Transform of the spectral density. Bardet et al. (2003) describe a concrete simulation procedure based on this method that overcomes some of the implementation issues encountered in practice. The procedure is:

1. Choose an even integer $M$. Define the vector of the Fourier frequencies $\Omega=\left(\theta_{1}, \ldots, \theta_{M / 2}\right)$, where $\theta_{t}=2 \pi t / M$ and compute the vector $F=f_{H}\left(\theta_{1}\right), \ldots, f_{H}\left(\theta_{M / 2}\right)$, where

$$
f_{H}(\theta)=\frac{1}{\pi} \sin (\pi H) \Gamma(2 H+1)(1-\cos \theta) \sum_{t \in \aleph}|2 \pi t+\theta|^{-2 H-1}
$$

$f_{H}(\theta)$ is the spectral density of fGn.

2. Generate $M / 2$ i.i.d exponential $\operatorname{Exp}(1)$ random variables $E_{1}, \ldots, E_{M / 2}$ and $M / 2$ i.i.d uniform $U[0,1]$ random variables $U_{1}, \ldots, U_{M / 2}$. 
3. Compute $Z_{t}=\exp \left(2 i \pi U_{t}\right) \sqrt{F_{t} E_{t}}$, for $t=1, \ldots, M / 2$.

4. Form the $M$-vector: $\tilde{Z}=\left(0, Z_{1}, \ldots Z_{(M / 2)-1}, Z_{M / 2}, \bar{Z}_{(M / 2)-1}, \ldots, \bar{Z}_{1}\right)$.

5. Compute the inverse FFT of the complex $Z$ to obtain the simulated sample path.

\subsubsection{Simulation of Fractional Stable Noise}

Replacing the integral in equation (61) with a Riemann sum, Stoev and Taqqu (2004) generate the approximation of fractional stable noise. They introduce parameters $n, N \in \aleph$, then express the fractional stable noise $Y(t)$ as

$$
Y_{n, N}(t):=\sum_{j=1}^{n N}\left(\left(\frac{j}{n}\right)_{+}^{H-1 / \alpha}-\left(\frac{j}{n}-1\right)_{+}^{H-1 / \alpha}\right) L_{\alpha, n}(n t-j)
$$

where $L_{\alpha, n}(t):=M_{\alpha}((j+1) / n)-M_{\alpha}(j / n), j \in \Re$. The parameter $n$ is mesh size and the parameter $M$ is the cut-off of the kernel function.

Stoev and Taqqu (2003) describe an efficient approximation involving the Fast Fourier Transformation (FFT) algorithm for $Y_{n, N}(t)$. Consider the moving average process $Z(m), m \in \aleph$,

$$
Z(m):=\sum_{j=1}^{n M} g_{H, n}(j) L_{\alpha}(m-j)
$$

where

$$
g_{H, n}(j):=\left(\left(\frac{j}{n}\right)^{H-1 / \alpha}-\left(\frac{j}{n}-1\right)_{+}^{H-1 / \alpha}\right) n^{-1 / \alpha}
$$

and where $L_{\alpha}(j)$ is the series of i.i.d standard stable Paretian random variables. Since $L_{\alpha, n}(j) \stackrel{d}{=}$ $n^{-1 / \alpha} L_{\alpha}(j), j \in \Re$, equation (74) and (75) imply $Y_{n, N}(t) \stackrel{d}{=} Z(n t)$, for $t=1, \ldots, T$. Then, the computing is moved to focus on the moving average series $Z(m), m=1, \ldots, n T$. Let $\tilde{L}_{\alpha}(j)$ be the $n(N+T)$-periodic with $\tilde{L}_{\alpha}(j):=L_{\alpha}(j)$, for $j=1, \ldots, n(N+T)$ and let $\tilde{g}_{H, n}(j):=g_{H, n}(j)$, for $j=1, \ldots, n N ; \tilde{g}_{H, n}(j):=0$, for $j=n N+1, \ldots, n(N+T)$, then

$$
\{Z(m)\}_{m=1}^{n T} \stackrel{d}{=}\left\{\sum_{j=1}^{n(N+T)} \tilde{g}_{H, n}(j) \tilde{L}_{\alpha}(n-j)\right\}_{m=1}^{n T}
$$

because for all $m=1, \ldots, n T$, the summation in equation (75) involves only $L_{\alpha}(j)$ with indices $j$ in the range $-n N \leq j \leq n T-1$. Using a circular convolution of the two $n(N+T)$-periodic series $\tilde{g}_{H, n}$ and $\tilde{L}_{\alpha}$ computed by using their Discrete Fourier Transforms (DFT), the variables $Z(n), m=1, \ldots, n T$ (i.e., the fractional stable noise) can be generated.

\subsection{Implications of Fractal Processes}

Fractal processes have been applied to the study of computer networks. Leland et al. (1994) and Willinger et al. (1997) employ fractal processes in modeling Ethernet traffic. Feldmann et al. (1998) discuss the fractal processes in the measurement of TCP/IP and ATM WAN traffic. Paxson and Floyd 
(1995), Paxson (1997), and Feldmann et al. (1998) discuss the characteristics of self-similarity in widearea traffic with respect to the fractal processes. Crovella and Bestavros (1997) provide evidence of selfsimilarity in world-wide web traffic by means of fractal processes modeling. An extensive bibliographical review of the research in the area of network traffic and network performance involving the fractal processes and self-similarity is provided by Willinger et al. (1996). Sahinoglu and Tekinay (1999) survey studies on the self-similarity phenomenon in multimedia traffic and its implications in network performance.

Baillie (1996) provides a survey of the major econometric research on long-range dependence processes, fractional integration, and applications in economics and finance. Bhansali and Kokoszka (2006) review recent research on long-range dependence time series. Recent theoretical and empirical research on long-range dependence in economics and finance is provided by Rangarajan and Ding (2006) and Teyssiére and Kirman (2006).

Based on the modeling mechanism of fractal processes, Sun et al. (2006) empirically compare fractional stable noise with several alternative distributional assumptions in either fractal form or i.i.d. form (i.e., normal distribution, fractional Gaussian noise, generalized extreme value distribution, generalized Pareto distribution, and stable distribution) for modeling returns of major German stocks. The empirical results suggest that fractional stable noise dominate these alternative distributional assumptions both in in-sample modeling and out-of-sample forecasting. This finding suggests that the model built on non-Gaussian non-random walk (fractional stable noise) performs better than those models based on either the Gaussian random walk, the Gaussian non-random walk, or the non-Gaussian random walk.

\section{Summary}

In this paper, we review recent studies in finance that use intra-daily price and return data. A large body of research confirms stylized facts (for example, random durations, heavy tailedness, seasonality, and long-range dependence) based on intra-daily data from both the equity market and the foreign exchange market. We summarize the stylized facts reported in the intra-daily data literature. We discuss the research fields in finance where the use of intra-daily data have attracted increased interest and potential applications, such as stylized facts modeling, market volatility and liquidity analysis, and market microstructure theory.

Recent research shows that intra-daily data exhibit strong dependence structure. In order to pull together long-range dependence study with intra-daily analysis, we briefly introduce research on longrange dependence and fractal processes. The principal problem in long-range dependence modeling is measuring the self-similar coefficient, i.e., the Hurst index. We review the studies on long-range dependence from three viewpoints: in the time domain, in the frequency domain, and with respect to econometric models. Modeling long-range dependence in intra-daily data with the help of fractal processes is introduced. 


\section{References}

[1] Adams, G., G. McQueen, and R. Wood (2004). The effects of inflation news on intra-daily sotck returns. Journal of Business, vol. 77, No. 3, pp. 547-574.

[2] Aït-Sahalia, Y., P. Mykland, and L. Zhang (2005). How often to sample a continuous-time process in the presence of market microstructure noise. Review of Financial Studies, vol. 18, pp. 356-416.

[3] Alexander, C. (2001). Market Models: A Guide to Financial Data Analysis, John Wiley \& Sons.: New York.

[4] Andersen, T. G., and T. Bollerslev (1997). Intraday periodicity and volatility persistence in financial markets. Journal of Empirical Finance, vol. 4, pp. 115-158.

[5] — (1998). Answering the skeptics: yes, standard volatility models do provide accurate forecasts. International Economic Review, vol. 39, pp. 885-905.

[6] Andersen, T. G., T. Bollerslev, and A. Das (2001). Variance-ratio statistics and high-frequency data: testing for changes in intraday volatility patterns. Journal of Finance, vol. 59, No. 1, pp. 305-327.

[7] Andersen, T. G., T. Bollerslev, F. X. Diebold, and H. Ebens (2001a). The distribution of realized stock return volatility. Journal of Financial Economics, vol. 61, pp. 43-76.

[8] Andersen, T. G., T. Bollerslev, F. X. Diebold, and P. Labys (2000). Exchange rate returns standardized by realized volatility are (nearly) Gaussian. Multinational Finance Journal, vol. 4, pp. 159-179.

[9] (2001b). The distribution of realized exchange rate volatility. Journal of the American Statistical Association, vol. 96 , pp. 42-55.

[10] _ (2003). Modeling and forecasting realized volatility. Econometrica, vol. 71, pp. 529-626.

[11] Andersen, T. G., T. Bollerslev, and F.X. Diebold (2005a), Parametric and nonparametric measurements of volatility, in Y. Ait-Sahalia and L.P.Hansen, eds., Handbook of Financial Econometrics, North Holland Press: Amsterdam

[12] Andersen, T. G., T. Bollerslev, P. F. Christoffersen, and F.X. Diebold (2006). Volatility and correlation forecasting, in G. Elliott, C. Granger, and A. Timmermann, eds.,Handbook of Economic Forecasting, North Holland Press: Amsterdam.

[13] - (2005b). Practical volatility and correlation modeling for financial market risk management, in M. Carey and R. Stulz, eds., Risks of Financial Institutions, University of Chicago Press.

[14] Baillie, R. T. (1996). Long memory processes and fractional integration in econometrics. Journal of Econometrics, vol. 73 , pp. 5-59.

[15] - (1990). Intra-day and inter-market volatility in foreign exchange rates. Review of Economic Studies, vol. 58, pp. 565-585.

[16] - and H. O. A. Mikkelsen (1996). Fractional integrated generalized autoregressive conditional heteroskedasticity. Journal of Econometrics, vol. 74, pp. 3-30.

[17] Banerjee, A., and G. Urga (2005). Modeling structural breaks, long memory and stock market volatility: an overview. Journal of Econometrics, vol. 129, pp. 1-34.

[18] Bardet, J., G. Lang, G. Oppenheim, A. Philippe, and M. Taqqu (2003). Generators of long-range dependent processes: a survey, In Theory and Applications of Long-range Dependence, P. Doulkhan, G. Oppenheim, and M. Taqqu (eds.). Birkhäuser: Boston.

[19] Barndorff-Nielsen, O. E., and N. Shephard (2002). Econometric analysis of realized volatility and its use in estimating stochastic volatility models. Journal of Royal Statistical Society B, vol. 64, No. 2, pp. 253-280. 
[20] Bauwens, L., and D. Veredas (2004). The stochastic conditional duration model: a latent variable model for the analysis of financial durations. Jouranl of Econometrics, vol. 119, pp. 381-412.

[21] Bauwens, L., and P. Giot (2000). The logarithmic ACD model: an application to the bid-ask quote process of three NYSE stocks. Annales d'Economie et de Statistique, vol. 60, pp. 117-149.

[22] — (2001). Econometric Modelling of Stock Market Intraday Activity. Kluwer Academic Publishers

[23] (2003). Asymmetric ACD models: introducing price information in ACD models. Empirical Economics, vol. 28, No. 4, pp. 709-731.

[24] Beltratti, A., and C. Morana (1999). Computing value at risk with high frequency data. Journal of Empirical Finance, vol. 6, pp. 431-455.

[25] Beran, J. (1994). Statistics for Long-Memory Processes, Chapman \& Hall: New York.

[26] Bessembinder, H. and K. Venkataraman (2004). Does an electronic stock exchange need an upstairs market? Journal of Financial Economics, vol. 73, pp. 3-36.

[27] Bhansali, R. J., and P. S. Kokoszaka (2006). Prediction of long-memory time series: a tutorial review, in Processes with Long-Range Correlations: Theory and Applications, G. Rangarajan and M. Ding, eds., Springer: New York.

[28] Blair, B. J., S. H. Poon, and S. J. Taylor (2001). Forecasting S\& P 100 volatility: the incremental information content of implied volatilities and high-frequency index returns. Journal of Econometrics, vol.105, pp.5-26.

[29] Bloomfield, R., and M. O'Hara (1999). Market transparency: who wins and who loses? Review of Financial Studies, vol. 2, No. 1, pp. 5-35.

[30] - (2000). Can transparent markets survive? Journal of Financial Economics, vol. 55, No. 3, pp. $425-459$.

[31] Boehmer, E., G. Saar, and L. Yu (2005). Lifting the veil: an analysis of pre-trade transparency at the NYSE. Journal of Empirical Finance, vol. 10, pp. 321-353.

[32] Bollen, B., and B. Inder (2002). Estimating daily volatility in financial markets utilizing intraday data. Journal of Empirical Finance, vol. 9, pp. 551-562.

[33] Bollen, N. P. B., T. Smith, and R. E. Whaley (2004). Modeling the bid/ask spread: measuring the inventory holding premium. Journal of Finance, vol. 60, No. 2, pp. 97-141.

[34] Bollerslev, T., R. Chou, and K. F. Kroner (1992). ARCH modeling in finance: A review of the theory and empirical evidence. Journal of Econometrics, vol. 52, pp. 783-815.

[35] Bollerslev, T., and I. Domowitz (1993). Trading patterns and prices in the interbank foreign exchange market. Journal of Finance, vol. 48, pp. 1421-1443.

[36] Bollerslev, T., and H. O. A. Mikkelsen (1996). Modelling and pricing long-memory in stock market volatility. Journal of Econometrics, vol. 73, pp. 154-184.

[37] Bollerslev, Tim, J. Cai, and F. M. Song (2000). Intraday periodicity, long memory volatility and macroeconomic announcement effects in the US treasury bond market. Journal of Empirical Finance, vol. 7, pp. 37-55.

[38] Bollerslev, T., and J. H. Wright (2001). High-frequency data, frequency domain inference and volatility forecasting. The Review of Economics and Statistics, vol. 83, No. 4, pp. 596-602.

[39] Bolerslev, T., and Y. B. Zhang (2003). Measuring and modeling systematic risk in factor pricing models using high-frequency data. Journal of Empirical Finance, vol. 10, pp. 533-558.

[40] Breidt, F. J., N. Crato, and P. de Lima (1998). The detection and estimation of long memory in stochastic volatility. Journal of Econometrics, vol. 73, pp. 325-348. 
[41] Chordia, T., R. Roll, and A. Subrahmanyam (2002). Order imbalance, liquidity and market returns. Journal of Financial Economics, vol. 65, pp. 111-130.

[42] Chung, K. H., and B. F. Van Ness (2001). Order handling rules, tick size and the intraday pattern of bid-ask spreads for Nasdaq stocks. Journal of Financial Markets, vol. 4, pp. 143-161.

[43] Corsi, F., U. Kretschmer, S. Mittnik, and C. Pigorsch (2005). The volatility of realized volatility. CFS Working Paper No.2005/33.

[44] Coughenour, J. F., and D. N. Deil (2002). Liquidity provision and the organizational form of NYSE specialist firms. Journal of Finance, vol. 57, No. 2, pp. 841-869.

[45] Coval, J. D., and T. Shumway (2001). Is sound just noise? Journal of Finance, vol. 56, No. 5, pp. 1887-1910.

[46] Crovella, M. E., and A. Bestavros (1997). Self-similarity in World Wide Web traffic: evidence and possible causes. IEEE/ACM Transactions on Networking, vol. 5, No. 6, pp. 835-846.

[47] Dacorogna, M., R. Gençay, U. A. Müller, R. B. Olsen, and O. V. Pictet (2001). An Introduction of High-Frequency Finance, Academic Press: San Diego.

[48] Diebold, F. X., and A. Inoue (2001). Long memory and regime switching. Journal of Econometrics, vol. 105, pp. 131-159.

[49] Doukhan, P., G. Oppenheim, and M. S. Taqqu (2003). Theory and Applications of Long-Range Dependence, eds., Birkhäuser: Boston.

[50] Dufour, A., and R. Engle (2000). Time and the price impact of a trade. Journal of Finance, vol. 55, No. 6, pp. $2467-2498$.

[51] Easley, D., and M. O'Hara (2003). Microstructure and asset pricing, in Handbook of the Economics of Finance, G. M. Constantinides, M. Harris, and R. Stulz, eds., Elsevier: North Holland. vol.47, pp.577-605

[52] Engle, R. (1982). Autoregressive conditional heteroskedasticity with estimates of the variance of U.K. inflation. Econometrica, vol. 50, No. 4, pp. 987-1008.

[53] — (2000). The econometrics of ultra-high frequency data. Econometrica, vol. 68, No. 1, pp. 1-22.

[54] - and J. R. Russell (1998). Autoregressive conditional duration: a new model for irregularly spaced transaction data. Econometrica, vol. 66, No. 5, pp. 1127-1162.

[55] — (2004). Analysis of high frequency data, in Y. Ait-Sahalia and L.P.Hansen, eds., Handbook of Financial Econometrics, North Holland Press: Amsterdam.

[56] Faust, J., J. H. Rogers, E. Swanson, and J. H. Wright (2003). Identifying the effects of monetary policy shocks on exchange rates using high frequency data. Journal of European Economic Association, vol. 1, No. 5, pp. 1031-1057.

[57] Feldmann, A., A. C. Gilbert, and W. Willinger (1998). Data networks as cascades: investing the multifractal nature of Internet WAN traffic. ACM Computer Communication Review, vol. 28, pp. 42-55.

[58] — , and T. G. Kurtz (1998). The changing nature of network traffic: scaling phenomena. ACM Computer Communication Review, vol. 28, pp. 5-29.

[59] Feng, D., G. J. Jiang, and P. Song (2004). Stochastic conditional duration models with "leverage effect" for financial transaction data. Journal of Financial Econometrics, vol. 119, pp. 413-433.

[60] Fernandes, M., and J. Gramming (2005). Nonparametric specification tests for conditional duration models. Journal of Econometrics, vol. 127, pp. 35-68.

[61] Fleming, J., C. Kirby, and B. Ostdiek (2003). The economic value of volatility timing using "realized" volatility. Journal of Financial Economics, vol. 67, pp. 47-509. 
[62] Franke, G., and D. Hess (2000). Information diffusion in electronic and floor trading. Journal of Empirical Finance, vol. 7 , pp. $455-478$.

[63] Gençay, R., F. Selçuk, and B. Whitcher (2001). Differentiating intraday seasonalities through wavelet multi-scaling. Physica A, vol. 289, pp. 543-556.

[64] - (2002). An Introduction to Wavelets and Other Filtering Methods in Finance and Economics, Elsevier: San Diego.

[65] Gençay, R., G. Ballocchi, M. Dacorogna, R. Olsen, and O. Pictet (2002). Real-time trading models and the statistical properties of foreign exchange rates. International Economic Review, vol. 42, No. 2, pp. 463-491.

[66] Gerhard, F., and N. Hautsch (2002). Volatility estimation on the basis of price intensities. Journal of Empirical Finance, vol. 9, pp. 57-89.

[67] Geweke, J., and S. Porter-Hudak (1983). The estimation and application of long memory time series models. Journal of Time Series Analysis, vol. 4, No. 4, pp. 221-238.

[68] Ghysels, E. (2000). Some econometric recipes for high-frequency data cooking. Journal of Business and Economic Statistics, vol. 18, No. 2, pp. 154-163.

[69] - C. Gouriéroux and, J. Jasiak (2004). Stochastic volatility duration models. Journal of Econometrics, vol. 119, pp. $413-433$.

[70] Giot, P., and S. Laurent (2004). Modeling daily Value-at-Risk using realized volatility and ARCH type models. Journal of Empirical Finance, vol. 11, pp. 379-398.

[71] Giraitis, L., and D. Surgailis (1990). A central limit theorem for quadratic forms in strongly dependent random variables and its application to asymptotical normality of Whittle's estimate. Probability Theory and Related Fields, vol. 86 , pp. 87-104.

[72] Gleason, K. C., I. Mathur, and M. A. Peterson (2004). Analysis of intraday herding behavior among the sector ETFs. Journal of Empirical Finance, vol. 11, pp. 681-694.

[73] Goodhard, C., and M. O'Hara (1997). High frequency data in financial market: issues and applications. Journal of Empirical Finance, vol. 4, pp. 73-114.

[74] Goodhard, C., and Richard Payne (2000). eds., The Foreign Exchange Market: Empirical Studies with High-Frequency Data, Palgrave Macmillan.

[75] Gouriéroux, C., and J. Jasiak (2001). Financial Econometrics: Problems, Models and Methods, Princetion University Press: Princeton and Oxford.

[76] Gramming, J., and K. O. Maurer (1999). Non-monotonic hazard functions and the autoregressive conditional duration model. The Econometrics Journal, vol. 3, pp. 16-38.

[77] Gwilym, O., and C. Sutcliffe (1999). High-Frequency Financial Market Data, Risk Books.

[78] Haldrup, N., and M. Nielsen (2006). A regime switching long memory model for electricity prices. Journal of Econometrics, vol. 135, pp. 349-376.

[79] Hannan, E. J. (1973). The asymptotic theory of linear time series models. Journal of Applied Probability, vol. 10, pp. 130-145.

[80] Harris, L. (2003). Trading and exchanges, Oxford university press: oxford.

[81] Hasbrouck, J. (1996). Modeling microstructure time series, in Maddala G., and C. R Rao, eds., Statistical Methods in Finance (Handbook of Statistics, Volume 14), North-Holland. 
[82] Haas, M., S. Mittnik, and M. Paolella (2004). Mixed normal conditional heteroskedasticity. Journal of Financial Econometrics, vol. 2, No. 2, pp. 211-250.

[83] Higuchi, T. (1988). Approach to an irregular time series on the basis of the fractal theory. Physica D, vol. 31, pp. $277-283$.

[84] Hong, H., and J. Wang (2000). Trading and returns under periodic market closures. Journal of Finance, vol. 55, No. 1, pp. 297-354.

[85] Hotchkiss, E. S., and T. Ronen (2002). The informational efficiency of the corporate bond market: an intraday analysis. The Review of Financial Studies, vol. 15, No. 5, pp. 1325-1354.

[86] Huang, R. D., and H. S. Stoll (2001). Tick size, bid-ask spreads, and market structure. Journal of Financial and Quantitative Analysis, vol. 36, No. 4, pp. 503-522.

[87] Hurst, H. (1951). Long-term storage capacity of reservoirs. Transactions of the American Society of Civil Engineers, vol.116, pp.770-808.

[88] Hurvich, C. M., and J. Brodeski (2001). Broadband semiparametric estimation of the memory parameter of a long memory time series using fractional exponential models. Journal of Time Series Analysis, vol. 22, No. 2, pp. 222-249.

[89] Jain, P.J., and G. Joh (1988). The dependence between hourly prices and trading volume. Journal of Financial and Quantitative Analysis, vol. 23, pp. 269-284.

[90] Jasiak, J. (1998). Persistence in intertrade durations. Finance, vol. 19, pp. 166-195.

[91] Kalay, A., O. Sade, and A. Wohl (2004). Measuring stock illiquidity: an investigation of the demand and supply schedules at the TASE. Journal of Financial Economics, vol. 74, pp. 461-486.

[92] Kolmogoroff, A. N. (1940). Wienersche Spiralen und einige andere interessante Kurven im Hilbertschen Raum. Comptes Rendus (Doklady) Acad. Sci. URSS (N.S.), vol. 26, pp. 115-118.

[93] Kryzanowski, L., and H. Zhang (2002). Intraday market price integration for shares cross-listed internationally. Journal of Financial and Quantitative Analysis, vol. 37, No. 2, pp. 243-269.

[94] Leland, W., M. Taqqu, W. Willinger, and D. Wilson (1994). On the self-similar nature of Ethernet traffic. IEEE/ACM Transactions on Networking, vol. 2, No. 1, pp. 1-15.

[95] Lamperti, J. (1962). Semi-stable stochastic Processes. Transactions of the American Mathematical Society, vol. 104, pp. 62-78.

[96] Lo, A. W. (1991). Long-term memory in stock market prices. Econometrica, vol. 59, pp. 1279-1313.

[97] Longstaff, F., and A. W. Wang (2004). Electricity forward prices: a high-frequency empirical analysis. Journal of Finance, vol. 59, No. 4 pp. 1977-1900.

[98] Madhavan, A. (2000). Market Microstructure: A survey. Journal of Financial Markets, vol. 3, pp. $205-258$.

[99] Maejima, M. (1983). On a class of self-similar processes. Zeitschrift für Vahrscheinlichkeitstheorie und verwandte Gebiete, vol.62, pp. 235-245.

[100] — , and S. Rachev (1987). An ideal metric and the rate of convergence to a self-similar process. Annals of Probability, vol.15, pp. 702-727.

[101] Mandelbrot, B. B. (1963). New methods in statistical economics. Journal of Political Economy, vol. 71, pp. 421-440.

[102] (1975). Limit theorems on the self-normalized range of weakly and strongly dependent processes. Zeitschrift für Wahrscheinlichkeitstheoie und verwandte Gebiete, vol. 31, pp. 271-285.

[103] (1983). The Fractal Geometry of Nature. Freeman: San Francisco. 
[104] — and J. R. Wallis (1969). Computer experiments with fractional Gaussian noises. Water Resources Research, vol. 5, pp. 228-267.

[105] Mandelbrot, B. B., and M. S. Taqqu (1979). Robust R/S analysis of long-run serial correlation. Bulletin of the International Statistical Institute, vol. 48, No. 2, pp. 69-104.

[106] Mansfield, P., S. Rachev, and G. Samorodnitsky (2001). Long strange segments of a stochastic process and long-range dependence. The Annals of Applied Probability. vol. 11, pp. 878-921.

[107] Marinelli, C., S. Rachev, R. Roll, and H. Göppl (2000). Subordinated stock price models: heavy tails and long-range dependence in the high-frequency Deutsche Bank price record, in Datamining and Computational Finance, G. Bol, G. Nakhaeizadeh, and K. Vollmer, eds., Physica-Verlag: Heidelberg.

[108] Marinucci, D., and P. M. Robinson (1999). Alternative forms of fractional Brownian motion. Journal of Statistical Planning and Inference, vol. 80, pp. 111-122.

[109] Martens, M. (2001). Forecasting daily exchange rate volatility using intraday returns. Journal of International Money and Finance, vol. 20, pp. 1-23.

[110] - Y. C. Chang, and S.J. Taylor (2002). A comparison of seasonal adjustment methods when forecasting intraday volatility. The Journal of Financial Research, vol. 25, No. 2 pp. 283-299.

[111] Masulis, R. W., and L. Shivakumar (2002). Does market structure affect immediacy of stock price responses to news? Journal of Financial and Quantitative Analysis, vol. 37, No. 4, pp. 617-648.

[112] McInish, T., and R. Wood (1992). An analysis of intradaily patterns in bid/ask spreads for NYSE stocks. Journal of Finance, vol. 47, pp. 753-746.

[113] Mittnik, S., M. S. Paolella, and S. T. Rachev. (2002). Stationary of stable power-GARCH processes. Journal of Econometrics, vol. 106, pp. 97-107.

[114] Morana, C., and A. Beltratti (2004). Structural change and long-range dependence in volatility of exchange rate: either, neither or both? Journal of Empirical Finance, vol. 11, pp. 629-658.

[115] Moulines, E., and P. Soulier (1999). Log-periodogram regression of time series with long-range dependence. Annals of Statistics, vol. 27, No. 4, pp. 1415-1439.

[116] Müller, U. A., M. Dacorogna, R. Olsen, O. Pictet, M. Schwarz, and C. Morgenegg (1990). Statistical study of foreign exchange rates, empirical evidence of a price change scaling law, and intraday analysis. Journal Banking and Finance, vol. 14, pp. 1189-1208.

[117] Müller, U. A., M. M. Dacorogna, R. D. Dave, R. Olsen, O. Pictet, and J. von Weizsacker (1997). Volatilities of different time resolutions: analyzing thedynamics of market components. Journal of Empirical Finance, vol. 4, pp. 213-239.

[118] Naik, N. Y., and P.K. Yadav (2003). Do dealer firms manage inventory on a stock-by-stock or a portfolio basis? Journal of Financial Economics, vol. 69, pp. 235-353.

[119] Nelson, D. B. (1991). Conditional heteroskedasticity in asset returns: a new approach. Econometrica, vol. 59, pp. $347-370$.

[120] Neilsen, B. E., and N. Shephard (2004). Econometric analysis of realized covariation: high frequency based covariance, regression, and correlation in financial economics. Econometrica, vol. 72, No. 3, pp. 885-925.

[121] O’Hara, M. (1995). Market Microstructure Theory, Blackwell: Cambridge.

[122] Paxson, V. (1997). Fast, approximation synthesis of fractional Gaussian noise for generating self-similar network traffic. Computer Communications Review, vol. 27, No. 5, pp. 5-18. 
[123] Peng, C. K., S. V. Buldyrev, M. Simons, H. E. Stanley, and A. L. Goldberger (1994). Mosaic organization of DNA nucleotides. Physical Review E, vol. 49, pp. 1685-1689.

[124] Peterson, M., and E. Sirri (2002). Order submission strategy and the curious case of marketable limit orders. Journal of Financial and Quantitative Analysis, vol. 37, No. 2, pp. 221-241.

[125] — and S. Mittnik (2000). Stable Paretian Models in Finance. Wiley: New York.

[126] Rachev, S. and G. Samorodnitsky (2001). Long strange segments in a long range dependent moving average. Stochastic Processes and their Applications, vol. 93, pp. 119-148.

[127] Rachev, S., C. Menn and F. Fabozzi (2005). Fat-Tailed and Skewed Asset Return Distributions. Wiley: New Jersey.

[128] Rangarajan, G., and M. Ding (2006). eds., Processes with Long-Range Correlations: Theory and Applications, Springer: New York.

[129] Robinson, P. M. (1991), Testing for strong serial correlation and dynamic conditional heteroskedasticity in multiple regression. Journal of Econometrics, vol. 47, pp. 67-84.

[130] (1994). Efficient tests of nonstationary hypotheses. Journal of the American Statistical Association, vol. 89, pp. 1420-1437.

[131] (1995a). Log periodogram regression of time series with long range dependence. The Annals of Statistics, vol. 23, No. 3, pp. 1048-1072.

[132] (1995b). Gaussian semiparametric estimation of long range dependence. The Annals of Statistics, vol. 23, No. 5, pp. $1630-1661$.

[133] — (2003). Time Series with Long Memory, eds., Oxford University Press: New York.

[134] Samorodnitsky, G. (1994). Possible sample paths of self-similar alpha-stable processes, Statistics and Probability Letters, vol. 19, pp. 233-237.

[135] - (1996). A class of shot noise models for financial applications, In Proceeding of Athens International Conference on Applied Probability and Time Series. Volume 1: Applied Probability, C. Heyde, Yu. V. Prohorov, R. Pyke, and S. Rachev, (eds.), Springer: Berlin.

[136] — (1998). Lower tails of self-similar stable processes. Bernoulli, vol.4, pp. 127-142.

[137] — , and M. S. Taqqu (1994). Stable Non-Gaussian Random Processes: Stochastic Models with Infinite Variance, Chapman \& Hall/CRC: Boca Raton.

[138] Schwartz, R. A., and R. Francioni (2004). Equity Markets in Action, John Wiley \& Sons, Inc: Hoboken.

[139] Smith, B. F., D. S. Turnbull, and R.W. White (2001). Upstairs market for principal agency trades: analysis of adverse information and price effects. Journal of Finance, vol. 56, No. 5, pp. 1723-1746.

[140] Stoev, S., V. Pipiras and M. Taqqu (2002). Estimation of the self-similarity parameter in linear fractional stable motion. Signal Processing, vol.82, pp. 1873-1901.

[141] Stoev, S., and M. Taqqu (2004). Simulation methods for linear fractional stable motion and FARIMA using the Fast Fourier Transform. Fractals, vol.12, No.1, pp. 95-121.

[142] Stoyanov, S., and B. Racheva-Iotova (2004a). Univariate stable laws in the field of finance-approximation of density and distribution functions. Journal of Concrete and Applicable Mathematics, vol.2, No.1, pp. 38-57.

[143] - (2004b). Univariate stable laws in the field of finance-parameter estimation. Journal of Concrete and Applicable Mathematics, vol.2, No.4, pp. 24-49. 
[144] - (2004c). Numerical methods for stable modeling in financial risk management. In Handbook of Computational and Numerical Methods, S. Rachev (ed). Birkhäuser: Boston.

[145] Spulber, D. F. (1999). Market Microstructure: Intermediaries and the Theory of the Firm, Cambridge University Press: Cambridge.

[146] Sun, W., Z. Rachev, and F. Fabozzi (2006a). Fratals or i.i.d.: evidence of long-range dependence and heavy tailedness form modeling German equity market volatility. Technical Report, University of Karlsruhe and UCSB.

[147] — , and P. Kalev (2006b). Fratals in duration: capturing long-range dependence and heavy tailedness in modeling trade duration. Technical Report, University of Karlsruhe and UCSB.

[148] Taqqu, M. S., and V. Teverovsky (1998). Estimating long-range dependence in finite and infinite variance sereis, in R. Adler, R. Feldman, and M. S. Taqqu, eds., A Practical Guide to Heavy Tails, Birkhäuser: Boston.

[149] — , and W. Willinger (1995). Estimators for long-range dependence: an empirical study, Fractals, vol. 3, No. 4, pp. 785-798.

[150] Taylor, S., and X. Xu (1997). The incremental volatility information in one million foreign exchange quotations. Journal of Empirical Finance, vol. 4, pp. 317-340.

[151] Teverovsky, V., and M. S. Taqqu (1995). Testing for long-range dependence in the presence of shifting means or a slowly declining trend using a variance-type estimator. Preprint.

[152] - and W. Willinger (1999). A critical look at Lo's modified R/S statistic., Journal of Statistical Planning and Inference, vol. 80, pp. 211-227.

[153] Teyssiére, G., and A. P. Kirman (2006). Long Memory in Economics, eds,, Springer: Berlin.

[154] Theissen, E. (2002). Price discovery in floor and screen trading systems. Journal of Empirical Finance, vol. 9, pp. 455-474.

[155] Thomakos, D. D, and Tao Wang (2003). Realized volatility in the futures markets. Journal of Empirical Finance, vol. 10, pp. 321-353.

[156] Tsay, R. S. (2002). Analysis of Financial Time Series. John Wiley \& Sons, Inc: New York.

[157] Velasco, C., and P. M. Robinson (2000). Whittle pseudo-maximum likelihood estimation for nonstationary time series. Journal of the American Statistical Association, vol. 95, pp. 1229-1243.

[158] Veredas, D., J. M. Rodrìguez-Poo, and A. Espasa (2002). On the (intraday) seasonality of a financial point process: a semiparametric approach. Working Paper, CORE DP 2002/23, Université catholique de Louvain.

[159] Wasserfallen, W., and H. Zimmermann (1985). The behavior of intradaily exchange rates, Journal of Banking and Finance, vol. 9, pp. 55-72

[160] Weston, J. P. (2002), Electronic communication networks and liquidity on the Nasdaq. Journal of Financial Services Research, vol. 22, pp. 125-139.

[161] Whittle, R. (1951). Hypothesis Testing in Time Series Analysis, Uppsala: Almqvist.

[162] Willinger, W., M. Taqqu, and A. Erramilli (1996). A bibliographical guide to self-similar traffic and performance modeling for modern high-speed networks. Stochastic Networks: Theory and Applications, Royal Statistical Society Lecture Notes Series, Vol.4, Oxford University Press.

[163] Willinger, W., M. Taqqu, R. Sherman, and D. Wilson (1997). Self-similarity through high-variability: statistical analysis of Ethernet LAN traffic at the source level. IEEE/ACM Transactions on Networking, vol. 5, No. 1, pp. $71-86$. 
[164] Willinger, W., V. Paxson, and M. Taqqu (1998). Self-similarity and heavy tails: structural modeling of network traffic . In A Practical Guide to Heavy Tails, R. Adler, R. Feldman, and M. Taqqu (eds.). Birkhäuser: Boston.

[165] Wood, R. A., T. H. McInish and J. K. Ord (1985). An investigation of transaction data for NYSE stocks. Journal of Finance, vol. 40, No. 3, pp. 723-739.

[166] Zhang, M., J. Russell, and R. Tsay (2001). A nonlinear autoregressive conditional duration model with applications to financial transaction data. Journal of Econometrics, vol. 104, pp. 179-207.

[167] Zumbach, G. O., and U. Müller (2001). Operators on inhomogeneous time series. International Journal of Theoretical and Applied Finance, vol. 4, No. 1, pp. 147-178. 